\title{
Die Übernahme befristet Beschäftigter im Kontext betrieblicher Personalpolitik ${ }^{1}$
}

\author{
Von Christian Hohendanner und Hans-Dieter Gerner
}

Zusammenfassung: Die vorliegende Studie leistet einen Beitrag zur Debatte um die Erosion der Normalarbeit. Wir untersuchen mit Daten des IAB-Betriebspanels unter welchen Voraussetzungen Übernahmen in unbefristete Verträge realisiert werden. Einerseits dienen befristete Arbeitsverträge den Betrieben dazu, ein adäquates Verhältnis zwischen Stabilität und Flexibilität zu erzielen. Übernahmen erfolgen in diesem Fall seltener. Andererseits werden Befristungen bei der Rekrutierung von Arbeitskräften eingesetzt. Dabei spielen die Verhandlungsoptionen der Vertragsparteien eine wichtige Rolle: Hat der Betrieb Schwierigkeiten, Fachkräfte zu rekrutieren oder zu halten und offene Stellen zu besetzen, sind Übernahmen wahrscheinlicher. In den überwiegend öffentlichen und nichterwerbsorientierten sozialen Dienstleistungseinrichtungen erfüllen Befristungen vor allem eine Flexibilitätsfunktion. Hohe Befristungsquoten stehen dort geringen Übernahmechancen gegenüber. Im produzierenden Gewerbe hat die Rekrutierungsfunktion hingegen eine größere Bedeutung. Insgesamt deutet wenig darauf hin, dass der internationale Wettbewerb direkt zum Anstieg befristeter Verträge beiträgt.

\section{Einleitung}

Seit den 80er Jahren ist angesichts der hohen Massenarbeitslosigkeit von der „Krise der Arbeitsgesellschaft“ (Dahrendorf 1980) oder gar dem unausweichlichen „Ende der Erwerbsarbeit" (Rifkin 1995) die Rede. Eng damit verbunden ist die Ansicht, dass das deutsche Normalarbeitsverhältnis zunehmend zugunsten von atypischen Beschäftigungsformen an Bedeutung verlöre (Mückenberger 1985). Im Kontext der Globalisierung (Blossfeld et al. 2008) führe ein erhöhter Flexibilitätsbedarf der Betriebe - flankiert durch Deregulierungsmaßnahmen des Staates - dazu, dass ,flexible' Arbeitsverhältnisse wie Leiharbeit oder befristete Arbeitsverträge sozial abgesicherte Daueranstellungen zunehmend ablösen.

Empirische Studien zur Beschäftigungsstabilität sind in Bezug auf eine etwaige Erosion der Normalarbeit allerdings zurückhaltend: In allen OECD Ländern ist der Anteil langfristiger Beschäftigungsverhältnisse nach wie vor hoch, sodass allenfalls von einem neuen Gleichgewicht zwischen flexiblen und stabilen Beschäftigungsverhältnissen gesprochen werden kann (Auer 2006). ${ }^{2}$ „Stabile“ Beschäftigung beginnt jedoch häufig mit einem befristeten Vertrag: Im Jahr 2007 lag der Anteil der befristeten Verträge an allen Neueinstellungen nach Angaben des IAB-Betriebspanels bei 45 Prozent (Tabelle 2). Im Jahr 2001 waren es lediglich 32 Prozent (vgl. Bellmann et al. 2009). Somit lässt sich unterhalb der Oberfläche des statistisch gemessenen Anteils langfristiger Beschäftigungsverhältnisse keineswegs von Kontinuität der Beschäftigungsstrukturen sprechen. Aufgrund der zunehmenden Bedeutung von Befristungen liefern Übergangsanalysen eine wichtige Grundlage für arbeitsmarktpolitische Bewertungen. Die Studie geht daher der Frage nach, in welchem Ausmaß und in welchen Betrieben und Branchen der Übergang gelingt und befristete Verträge eine Brückenfunktion in stabile Beschäftigung erfüllen.

1 Wir danken den anonymen Gutachtern sowie Martin Abraham, Lutz Bellmann, Stefan Fuchs, Johannes Giesecke, Stefanie Gundert, Martin Heidenreich und Ulrich Walwei für wertvolle Hinweise.

2 Diese ,statistische' Stabilität bedeutet allerdings nicht, dass in qualitativer Hinsicht keine Veränderungen zu beobachten sind. Die zunehmende Unsicherheit strahlt etwa über die Präsenz atypisch Beschäftigter in den Betrieben oder die Verkürzung der Bezugsdauer des Arbeitslosengeldes auch auf die Mittelschicht der unbefristet Beschäftigten aus (vgl. Dörre 2007; Bosch et al. 2007).

Soziale Welt 61 (2010), S. $27-50$ 
Die zahlreichen bisherigen empirischen Analysen zu befristeten Arbeitsverhältnissen in Deutschland auf Basis von Personendaten zeigen, dass die Besetzung befristeter Stellen und die Übergänge in unbefristete Stellen in systematischer Weise erfolgen. ${ }^{3}$ Insbesondere Berufsanfänger und ältere Arbeitnehmer sowie Beschäftigte ohne abgeschlossene Berufsausbildung und Hochschulabsolventen werden häufig befristet (Gundert 2007, Giesecke 2006, Rudolph 2005). Übergangsanalysen stellen fest, dass etwa 40 Prozent der befristet Beschäftigten nach einem Jahr ein unbefristetes Arbeitsverhältnis beim selben oder einem anderen Arbeitgeber gefunden haben (Boockmann / Hagen 2006) und etwa zwei Drittel der befristet Beschäftigten nach drei Jahren in Dauerstellen tätig sind (Giesecke 2006). Für etwa ein Drittel können Zeitverträge demnach zur Prekarisierung der Erwerbskarrieren beitragen. Während für gut qualifizierte Arbeitskräfte Chancen auf stabile Weiterbeschäftigung bestehen, haben Personen ohne Ausbildung oder infolge von Arbeitslosigkeit eher schlechte Chancen (Gundert 2007, McGinnity et al. 2005).

Aus betrieblicher Perspektive wurden in Deutschland bisher vor allem die Determinanten der Nachfrage befristeter Verträge untersucht (Pfeifer 2006; Fritsch / Schank 2005, Pfeifer 2005; Meyer / Pfeifer 2005; Boockmann / Hagen 2003; Hagen 2003; Hagen / Boockmann 2002; Kaiser / Pfeiffer, 2000). Auch hier zeigen sich vor allem branchen- und betriebsgrößenspezifisch systematische Unterschiede in der Befristungspraxis (Fritsch / Schank 2005). Befristungen können die Anpassungsgeschwindigkeit der Arbeitsnachfrage bei externen Schocks erhöhen (Hagen 2003) und werden als Flexibilitätspuffer genutzt (Pfeifer 2005). Nach Hagen / Boockmann (2002) haben sowohl der Kündigungsschutz als auch Betriebsräte einen positiven Einfluss auf den Einsatz befristeter Verträge.

Die empirische Analyse der Übernahme in unbefristete Beschäftigung mit Betriebsdaten steht jedoch noch aus. Übernahmen bieten sich an, die Entwicklung der Beschäftigungsformen zu untersuchen, da sie an der Schnittstelle zwischen atypischer und normaler Arbeit liegen. Durch die Betrachtung von Übernahmen lassen sich Anhaltspunkte finden, ob Befristungen eher als Rekrutierungs- und Screeninginstrument oder eher als Flexibilitätspuffer genutzt werden. Dies ist weder mit Analysen auf Basis von Personendaten noch mit Betriebsanalysen möglich, die das bloße Auftreten von Befristungen untersuchen. ${ }^{4}$ Die Untersuchungseinheit Betrieb ist insofern besonders geeignet, da die Betriebe als zentrale Verursacher atypischer Beschäftigung betrachtet und empirisch analysiert werden können. Da sich die Betriebe unterschiedlicher Sektoren in ihrer Befristungs- und Übernahmepraxis deutlich unterscheiden (vgl. Bellmann et al. 2009), werden zudem die branchenspezifischen Kontextfaktoren betrieblicher Personalpolitik herausgearbeitet. Dabei liegt der Fokus - entsprechend eines , most different designs “ - auf einer Gegenüberstellung der Einrichtungen des sozialen Dienstleistungssektors und Betrieben des produzierenden Gewerbes.

Zunächst werden allgemeine (Kapitel 2.1 und 2.2) und branchenspezifische Thesen (Kapitel 2.3) zum Zusammenhang zwischen betrieblicher Personalpolitik und den Übernahmen befristet Beschäftigter entwickelt. Kapitel 3 stellt die Datenbasis - das IAB-Betriebspanel 2005 bis 2007 - und die empirische Methode - ein Zero-Inflated Negative-Binomial Modell (ZINB) - vor. Im Ergebnisteil (Kapitel 4) werden Befristungsanteile, Einstellungs- und Übernahmequoten befristeter Verträge nach Branchen und Betriebsgrößen analysiert. Anschließend werden die Ergebnisse zu den betrieblichen Bestimmungsfaktoren der Übernahmen befristet Beschäftigter dargestellt: Insgesamt zeigt sich, dass die Zunahme befristeter Verträge kein sub-

3 Eine ausführlichere Darstellung zum aktuellen Forschungsstand findet sich z.B. bei Lengfeld und Kleiner (2009) oder Giesecke (2006).

4 Die jeweils einseitige Betrachtung von Befristungen mit Personen- oder Betriebsdaten ist dem Umstand geschuldet, dass keine verknüpften Arbeitnehmer-Arbeitgeber-Daten mit Informationen über befristete Arbeitsverhältnisse vorliegen. 
jektloser, etwa durch ,Globalisierung' hervorgerufener Prozess ist (vgl. Offe 2008), sondern abhängt von konkreter betrieblicher Personalpolitik, die zu großen Teilen bestimmt wird durch den (reformierbaren) institutionellen Kontext sowie durch die Verhandlungsposition der Arbeitsmarktakteure.

\section{Befristungen im Kontext betrieblicher Personalpolitik}

Während unbefristet Beschäftigte grundsätzlich selbst über die Beendigung ihres Arbeitsverhältnisses entscheiden können, gehen Befristungen mit einer Einschränkung von Handlungsoptionen einher. Nur wenige Arbeitnehmer willigen daher in befristete Arbeitsverträge aus freien Stücken ein und die unbefristete Vollzeitbeschäftigung dient nach wie vor als erstrebenswertes Ziel und Bezugspunkt: Der Anteil befristet Beschäftigter in Deutschland, die keine Dauerstellung wünschen, lag im Jahr 2006 nach Angaben der Europäischen Arbeitskräfteerhebung bei $2 \%$ (Bellmann et al. 2009). Die Verbreitung von befristeten Arbeitsverträgen geht also in erster Linie von den Betrieben aus. ${ }^{5}$

Ein zentrales betriebliches Funktionserfordernis in der Personalpolitik ist die Sicherung der Verfügbarkeit von Arbeitskräften. ${ }^{6}$ Zur deren Sicherstellung gehören die Beschaffung, Qualifizierung und Bindung von Personal (Köhler et al. 2007). Dabei lassen sich zwei Dimensionen unterscheiden: Erstens hat der Betrieb die Aufgabe der Rekrutierung und Qualifizierung, wobei beides intern und extern erfolgen kann (ebd.). Zweitens muss der Betrieb im Hinblick auf seine Personalpolitik mit Diskontinuität umgehen. Im Sinne des Transaktionskostenansatzes entsteht dauerhafte Beschäftigung durch die Häufigkeit und Dauer von Transaktionen bzw. des kontinuierlichen Bedarfs an bestimmten Qualifikationen. Umgekehrt erschweren Schwankungen von Auftrags- und Arbeitsvolumina und Budgetrestriktionen dauerhafte Beschäftigung (ebd.).

Im Folgenden wird der Einsatz von Befristungen und Übernahmen in unbefristete Verträge im Zusammenhang mit der Lösung des Verfügbarkeitsproblems diskutiert. Das Verfügbarkeitsproblem mit seinen beiden Dimensionen - Diskontinuität und Rekrutierung - wird daher im folgenden Abschnitt ausführlich dargestellt.

\subsection{Diskontinuitätsproblem und befristete Verträge}

Das Diskontinuitätsproblem betrifft einerseits die Umweltkontingenzen der Unternehmung (Wettbewerbssituation, schwankende Güternachfrage, Haushaltslage oder Arbeitsmarktsituation) und andererseits die Grenzen des Betriebs selbst. Die Herstellung eines möglichst günstigen Verhältnisses zwischen Stabilität und Anpassungsfähigkeit ist das Ziel der Kombination verschiedener Flexibilisierungsstrategien in der Personalpolitik (Struck 2006). Befristungen sind vor allem ein Anpassungsmittel der Arbeitsmenge im Umgang mit Diskontinuitäten (Pfeifer 2006; Pfeifer 2005; Meyer / Pfeifer 2005; Boockmann / Hagen 2003; Hagen 2003;

5 Als weiterer wichtiger Akteur ist der Staat zu nennen (Vielle / Walthery 2003): Die staatliche Deregulierung des Befristungsrechts bzw. die partielle Lockerung des Kündigungsschutzes wird einerseits mit der Bekämpfung der Arbeitslosigkeit begründet (Büchtemann 1990). Die vermuteten geringeren betrieblichen Kosten befristeter Verträge, sollen das Einstellungsrisiko für Betriebe verringern und die Integration in den Arbeitsmarkt erleichtern. Andererseits soll der Flexibilitätsspielraum der Betriebe erhöht werden. Der Staat erfüllt in diesem Zusammenhang allerdings nicht nur eine regulierende Funktion. Er ist als Arbeitgeber selbst in hohem Maße an der Verbreitung befristeter Verträge beteiligt (Giesecke 2006).

6 Die betrieblichen Funktionserfordernisse lassen sich in der Tradition industriesoziologischer und personalökonomischer Ansätze in Form von zwei betrieblichen Bezugsproblemen darstellen (Köhler et al. 2007): Verfügbarkeit und Leistungsbereitschaft. Bei der Sicherstellung der Leistungsbereitschaft geht es um Motivation, Kontrolle und Herrschaftssicherung. 
Hagen / Boockmann 2002; Kaiser / Pfeiffer 2001). ${ }^{7}$ Die Mengenanpassung durch Entlassungen ist aufgrund des Bestandsschutzes der Stammbelegschaft durch den allgemeinen Kündigungsschutz, das Mitbestimmungsrecht des Betriebsrats ${ }^{8}$ oder tarifvertragliche Schutzrechte an Bedingungen geknüpft. Insbesondere in Großbetrieben herrscht aufgrund der zahlreichen arbeitsrechtlichen Schwellenwerte (Koller et al. 2007) sowie der Existenz interner Arbeitsmärkte zum Teil ein erhöhter Kündigungsschutz. Die Umgehung des Bestandsschutzes ist ein inhärenter Grund für den Abschluss befristeter Verträge. Es konnte nachgewiesen werden, dass sowohl der Kündigungsschutz als auch die Existenz eines Betriebsrates einen positiven Einfluss auf den Einsatz befristet Beschäftigter ausüben (Pfeifer 2005; Hagen / Boockmann 2003). Befristungen erfüllen die Funktion, Ausfälle von Arbeitskräften (Krankheit, Mutterschaftsurlaub etc.) zu kompensieren sowie Entlassungskosten zu antizipieren, wenn eine dauerhafte Beschäftigung nicht sicher oder nicht beabsichtigt ist. Dies ist der Fall bei wirtschaftlicher Unsicherheit über die zukünftige Auftragslage oder bei (z.B. saisonal bedingten) Nachfrageschwankungen (Hagen / Boockmann 2002). ${ }^{9}$ In Betrieben, in denen umgekehrt der allgemeine Kündigungsschutz nicht gilt, liegt der Grund für eine Befristung vor allem im zeitlich begrenzten Bedarf der Arbeitskraft. Übernahmen sind in diesen Betrieben unwahrscheinlich. Ist der Bestandsschutz des Stammpersonals wiederum so stark ausgeprägt, dass Kündigungen kaum möglich sind, führt eine Personalpolitik zu ,perversen Effekten“ (Blanchard / Landier 2002), die de facto nur zwei Extrempole kennt: Unkündbarkeit auf geschlossenen Positionen und Befristungen. Auch in diesem Fall sind Übernahmen unwahrscheinlich. Für den Arbeitgeber bestehen Anreize, eher eine neue Arbeitskraft wiederum befristet einzustellen, als sich dauerhaft an eine Arbeitskraft zu binden, deren Verhandlungsmacht bei Übernahme zunehmen würde. Der heterogene Kündigungsschutz polarisiert demnach die Arbeitsmarktrisiken zwischen befristet und unbefristet Beschäftigten (Giesecke 2006). Die Übernahme von befristet Beschäftigten hingegen ist Ausdruck eines stabilen Mengenbedarfs des Betriebs und deutet darauf hin, dass wirtschaftliche Unsicherheiten, schwankende Aufträge oder eine mögliche Unkündbarkeit sowie andere Anpassungsprobleme einer dauerhaften Beschäftigung nicht im Wege stehen.

7 Grundsätzlich können Betriebe die Arbeitsmenge über die Angleichung von Arbeitszeiten und Beschäftigungsvolumina steuern. Darunter fallen Entlassungen und Einstellungen, atypische Beschäftigungsformen, flexible Arbeitszeiten (Teilzeit, Überstunden, Arbeitszeitkonten etc.), die Aus- und Eingliederung von Betriebsteilen oder die Verlagerung von Aufgaben an Externe.

8 Der Betriebsrat bedeutet für den Arbeitgeber aufgrund des Mitbestimmungsrechts bei Personalfragen eine Erhöhung des Kündigungsschutzes. Betriebsräte stellen einen Ausgleich der Machtasymmetrie dar. Ihre Aufgabe besteht darin, die Kernbelegschaft zu schützen; sie erhöhen damit die Verhandlungsmacht der Arbeitnehmer auf geschlossenen Positionen und befürworten den Schutz der Kernbelegschaft durch eine flexible Randbelegschaft (Boockmann / Hagen 2003). Die Stärkung der geschlossenen Positionen kann Arbeitgeber allerdings dazu drängen auf offene Positionen auszuweichen. Zugleich sind Betriebräte auch Vertreter befristet Beschäftigter und versuchen demzufolge einer Politik des hire-and-fire entgegenzuwirken. Sie fördern tendenziell „langfristig-interne Beschäftigungsstrategien“ (Nienhüser 2007). In Betrieben ohne Betriebsrat fehlt hingegen eine Instanz, die eine exzessive Nutzung atypischer Verträge bzw. die Machtasymmetrie zwischen Betrieb und Arbeitskräften begrenzt. Allerdings ist der Bestandsschutz der Kernbelegschaft in Betrieben ohne Betriebsrat tendenziell geringer und Arbeitgeberkündigungen kostengünstiger. Insofern sind die Wirkungen des Betriebsrats auf die Übernahme befristet Beschäftigter uneindeutig.

9 Eine zentrale Erklärung für die Existenz von befristeten Arbeitsverträgen liefert darüber hinaus das Kern-Peripherie-Modell: Teile der Belegschaft, deren Qualifikationen und Fähigkeiten entscheidend für den Betrieb und schwierig zu ersetzen sind, sind ein Teil der Kernressourcen, die vor Diskontinuitäten weitgehend geschützt werden. Anpassungen konzentrieren sich auf eine verzichtbare Randbelegschaft. Empirisch konnte die Kern-Peripherie-These allerdings bisher nicht belegt werden, auch da Schwierigkeiten bestehen, Kern- und Randbelegschaft in den Daten sauber zu trennen (Pfeifer 2005; Capelli / Neumark 2004). 
Diskontinuitätsthese: Werden befristete Arbeitsverträge zur Lösung des Diskontinuitätsproblems herangezogen, ist die Übernahmewahrscheinlichkeit in unbefristete Beschäftigung gering.

\subsection{Rekrutierungsproblem und befristete Verträge}

Eine zentrale Aufgabe betrieblicher Personalpolitik besteht darin, die Verfügbarkeit qualifizierter Arbeitskräfte für den Arbeits- und Produktionsprozess sicherzustellen. Dabei stellt sich die Frage, ob auf bereits extern qualifizierte Arbeitskräfte zurückgegriffen, selbst ausgebildet oder der Arbeitsprozess so gestaltet wird, dass keine besonderen Qualifikationen erforderlich sind. Für Deutschland finden diese betrieblichen Strategien idealtypisch ihre Entsprechung in einer dreiteiligen Segmentierung des Arbeitsmarktes in unstrukturierte, berufsfachliche und betriebsinterne Arbeitsmärkte (Lutz 1987; Sengenberger 1987). In internen Arbeitsmärkten mit hoher Arbeitsplatzsicherheit dienen befristete Verträge als ,ports of entry“ (Doeringer / Piore 1971). Aufgrund des dort vorhandenen hohen Bestandsschutzes kommt dem Screening der Mitarbeiter eine große Bedeutung zu. Übernahmen sind als erfolgreiches Ende einer Testphase geeigneter Mitarbeiter aufzufassen. Bisherige Studien zeigen für berufsfachliche Arbeitsmärkte, dass Arbeitnehmer mit beruflichen Ausbildungsabschlüssen ein geringeres Befristungsrisiko aufweisen als Hochschulabsolventen und Personen ohne abgeschlossene Berufsausbildung (McGinnity et al. 2005; Giesecke / Groß 2002). Dies kann zum Teil mit dem Abbau von Informationsasymmetrien während der Ausbildungszeit und der fehlenden Berufserfahrung von Hochschulabsolventen im Vergleich zu Azubis erklärt werden. Befristete Verträge werden demnach dazu genutzt, Defizite der Institutionalisierung des ,school-towork"-Übergangs auszugleichen. In dem Maße, in dem Informationsasymmetrien über die Eignung von Berufsanfängern bzw. von neuen Arbeitskräften bestehen, werden Befristungen als „Screening“-Instrument eingesetzt, um diese Informationsrückstände zu beheben, ohne zugleich das Risiko potenzieller Entlassungskosten zu übernehmen.

Screeningthese: Werden befristete Arbeitsverträge als Eignungstest eingesetzt, ist die Übernahmewahrscheinlichkeit in unbefristete Beschäftigung hoch. Übernahmen bedeuten demnach, dass die Arbeitskräfte auf Dauer den betrieblichen Anforderungen entsprechen.

Eine exzessive Befristungspraxis ist für den Betrieb mit Risiken verbunden. Beschäftigungsunsicherheit führt dazu, dass sich Arbeitnehmer eher einen Betrieb mit stabileren Bedingungen suchen, wenn die Optionen auf dem externen Arbeitsmarkt es zulassen (Ashford et al. 1989; Hellgren et al. 1999). Daraus folgt, dass gerade die produktiven Arbeitskräfte mit den besseren Arbeitsmarktchancen den Betrieb verlassen, werden sie nicht rechtzeitig übernommen. Besteht ein Wettbewerb um produktive, hochqualifizierte Arbeitskräfte, sind Betriebe bestrebt, die Arbeitskräfte über unbefristete Verträge zu halten bzw. zu übernehmen. Besteht hingegen ein Überhang an verfügbaren Arbeitskräften auf dem externen Arbeitsmarkt, sind angesichts der fehlenden Alternative der Arbeitnehmer - befristete Verträge für die Arbeitgeber mit geringen Risiken verbunden, leichter durchsetzbar und Übernahmen eher unwahrscheinlich. Bei entsprechendem Ungleichgewicht auf dem Arbeitsmarkt gilt dies auch für Beschäftigungssysteme, die dem Idealtypus des internen Arbeitsmarktes nahekommen: Betriebsspezifisch qualifizierte Arbeitskräfte werden zeitweise in der Arbeitslosigkeit geparkt und bei Bedarf reaktiviert (Liebig / Hense 2007).

Rekrutierungsthese: Wenn der Betrieb auf einen großen Arbeitskräftepool auf dem externen Arbeitmarkt für die jeweilige Tätigkeit zurückgreifen kann, ist die Übernahmewahrscheinlichkeit gering. Umgekehrt steigt die Übernahmechance bei einer besonderen Verhandlungsposition qualifizierter Arbeitskräfte. 


\subsection{Der branchenspezifische Kontext}

Die Zunahme des Bedarfs flexibler Beschäftigungsverhältnisse wird häufig mit der Globalisierung bzw. der beschleunigten Volatilität der Märkte begründet. Insofern müssten befristete Verträge zur Lösung des Diskontinuitätsproblems gerade im Produktionssektor ${ }^{10}$ eine große Rolle spielen. Hiergegen spricht einerseits, dass im Produktionssektor Flexibilität vor allem über interne Flexibilisierung (Hohendanner / Bellmann 2007) und im Segment der einfacheren Tätigkeiten über Leiharbeit garantiert wird (Bellmann et al. 2009). Andererseits stellt sich das Rekrutierungsproblem aufgrund der dominanten qualifikationsbasierten Produktmarktstrategie in besonderer Weise. Ein zentrales Element des deutschen Produktionssystems ist die Kombination berufsfachlicher und betriebsspezifischer Qualifikationen der Beschäftigten, die mit Humankapitalinvestitionen der Betriebe einhergehen und vor allem inkrementelle Produktinnovationen (Hall / Soskice 2001) auf hohem Niveau ermöglichen. Mit kurzfristigen Verträgen lässt sich das notwendige Humankapital weder aufbauen noch lassen sich die betrieblichen Investitionen amortisieren. Der allgemeine Kündigungsschutz als Teil des deutschen Institutionengefüges stellt für den Produktionssektor aus dieser Perspektive kein Hindernis dar, sondern unterstützt diese spezifisch deutschen Produktmarktstrategien. Das Zusammenspiel der Institutionen ist somit ein geeigneter Nährboden für Produkte etwa des Automobilsektors oder im Maschinenbau und geht mit komparativen Kostenvorteilen gegenüber Unternehmen aus anderen Ländern einher. Gerade im Produktionssektor und in exportorientierten Betrieben ist demzufolge zu erwarten, dass Befristungen eher als Screeninginstrument genutzt werden und das Interesse an langfristiger Beschäftigung dominiert.

These zum produzierenden Gewerbe: Betriebe des Produktionssektors setzen bei der Lösung des Diskontinuitätsproblems vor allem auf interne Flexibilisierung und - in geringerem Ausmaß - auf Leiharbeit. Befristungen werden überwiegend als Screeninginstrument genutzt. Die Übernahmechance ist dementsprechend hoch.

Die Situation des Produktionssektors mit seiner starken Exportorientierung ist nicht übertragbar auf den Dienstleistungssektor. Insbesondere im sozialen, ${ }^{11} \mathrm{zu}$ großen Teilen öffentlichen Dienstleistungssektor stellen sich die Kontextfaktoren der Personalpolitik völlig anders dar als in rein marktwirtschaftlich organisierten Wirtschaftszweigen. ${ }^{12}$ Die Funktion befristeter Arbeitsverträge im öffentlichen Dienst besteht im Sinne des Diskontinuitätsproblems einerseits in der Vermeidung des ausgeprägten Bestandschutzes der regulären Arbeitskräfte. Verstärkt wird die Befristungspraxis andererseits durch das Bestreben des Staates im Zuge der Verwaltungsmodernisierung öffentliche Haushalte zu konsolidieren und den Stellenbestand über ,inkrementelle Personalreduzierung“ (Jann et al. 2007) abzubauen (Giesecke 2006). Die Verwaltungsmodernisierung der 90er Jahre hat sich dabei immer stärker zu einer Außenmodernisierung der „Zulieferstrukturen“(Dahme / Wohlfahrt 2006) - den privaten, privatisierten

10 Wir unterscheiden dazu einerseits zwischen primärem (Land- und Forstwirtschaft), sekundärem (Produktion) und tertiärem Sektor (Dienstleistungen). Der Dienstleistungssektor wird entsprechend der OECD-Klassifikation (2000) zudem in vier Typen unterteilt - Produktionsorientierte Dienstleistungen, Distributive Dienstleistungen, Soziale Dienstleistungen und Personenbezogene Dienstleistungen. Produktionsorientierte und distributive Dienstleistungen richten sich vor allem an Produktions- und Vermarktungsaktivitäten des produzierenden Gewerbes, während personenbezogene und soziale Dienstleistungen verstärkt von privaten Haushalten konsumiert werden. Staatlich finanzierte oder geführte Betriebe sind vor allem im Bereich der sozialen Dienstleistungen zu finden.

11 Der soziale Dienstleistungssektor umfasst vor allem öffentliche, nicht-erwerbsorientierte und private Betriebe und Einrichtungen der Bereiche Erziehung und Unterricht, Gesundheit und Soziales, Kultur und Sport sowie die öffentliche Verwaltung (vgl. OECD 2000).

12 Die übrigen - äußerst heterogenen - Dienstleistungsbranchen zeichnen sich in der Befristungspraxis nicht durch ein klar erkennbares Muster aus. Auf eine detaillierte Analyse der Übernahmeproblematik weiterer Dienstleistungsbereiche wird daher verzichtet. 
und nichterwerbsorientierten Dienstleistern - weiterentwickelt. Soziale Dienstleistungen erfolgen zunehmend nach quasi-betriebswirtschaftlicher Effizienz- und Wettbewerbslogik über Kontraktmanagement bei gleichzeitiger Steuerungshoheit des Staates, d.h. Abhängigkeit von staatlicher Finanzierung und öffentlichem Leistungsrecht. Der hohe Anteil befristeter Arbeitsverträge im sozialen Dienstleistungsbereich kann als Ausdruck dieser quasi-marktlichen Steuerung gesehen werden. Der zunehmende Kostendruck sowie die zeitlich begrenzte Vergabe von öffentlichen Mitteln lassen sich zum Teil nur über befristete Arbeitsverträge kanalisieren. Befristungen erfüllen eher die Funktion eines Flexibilitätspuffers, die Übernahmechancen sind daher gering. Der Produktionssektor mag sich mit dem institutionellen Gefüge und dem Kündigungsschutz arrangieren bzw. Vorteile daraus ziehen. Betriebe im Sozialsektor können hingegen ihre Wettbewerbsfähigkeit nur erhalten, indem sie institutionelle Regulierungen umgehen - etwa durch den Einsatz befristeter Arbeitsverträge (vgl. Herrmann 2008).

These zu sozialen Dienstleistungen: Befristete Verträge werden in den (öffentlichen, privaten und nicht-erwerbsorientierten) sozialen Dienstleistungen vor allem zur Lösung des Diskontinuitätsproblems eingesetzt. Die Übernahmechance ist dementsprechend gering.

Tabelle 1: Thesen zur Übernahme befristet Beschäftigter in ein unbefristetes Arbeitsverhältnis

\begin{tabular}{|l|l|}
\hline Diskontinuitätsthese & $\begin{array}{l}\text { Werden befristete Verträge zur Lösung des Diskontinuitätsproblems her- } \\
\text { angezogen, ist die Übernahmewahrscheinlichkeit gering. }\end{array}$ \\
\hline Screeningthese & $\begin{array}{l}\text { Werden befristete Arbeitsverträge zum Abbau von Informationsasymme- } \\
\text { trien als Eignungstest bzw. als funktionales Äquivalent zu Ausbildungs- } \\
\text { verträgen eingesetzt, ist die Übernahmewahrscheinlichkeit in unbefristete } \\
\text { Beschäftigung hoch. Übernahmen bedeuten demnach, dass die Arbeits- } \\
\text { kräfte auf Dauer den betrieblichen Anforderungen entsprechen. }\end{array}$ \\
\hline Verfügbarkeitsthese & $\begin{array}{l}\text { Wenn der Betrieb auf einen großen Arbeitskräftepool auf dem externen } \\
\text { Arbeitmarkt für die jeweilige Tätigkeit zurückgreifen kann, ist die Über- } \\
\text { nahmewahrscheinlichkeit gering. }\end{array}$ \\
\hline Branchenspezifische Thesen \\
\hline Produktionssektor & $\begin{array}{l}\text { Betriebe des Produktionssektors erzielen Flexibilität über interne Flexibi- } \\
\text { lisierung und Leiharbeit und nutzen Befristungen überwiegend als Scree- } \\
\text { ninginstrument. Die Übernahmewahrscheinlichkeit ist dementsprechend } \\
\text { hoch. }\end{array}$ \\
\hline Soziale Dienstleistungen & $\begin{array}{l}\text { Befristete Verträge werden in den sozialen Dienstleistungen und im öf- } \\
\text { fentlichen Dienst vor allem zur Lösung des Diskontinuitätsproblems ein- } \\
\text { gesetzt. Der ,organisierte Wettbewerb“ sowie die Abhängigkeit von zeit- } \\
\text { lich befristeten öffentlichen Mitteln befördern eine exzessive Befristungs- } \\
\text { praxis und behindern Übernahmen. Die Übernahmewahrscheinlichkeit ist } \\
\text { dementsprechend niedrig. }\end{array}$ \\
\hline
\end{tabular}

\section{Daten und Methode}

Als Datenbasis für die folgende Analyse dient das IAB-Betriebspanel (zur Konzeption: Fischer et al. 2009; Bellmann 2002). Hierbei handelt es sich um eine jährlich wiederholte Betriebsbefragung von etwa 16.000 Betrieben aller Branchen und Betriebsgrößen, die seit 1993 für Westdeutschland und seit 1996 für Gesamtdeutschland im Auftrag des IAB von TNS Infratest Sozialforschung durchgeführt wird. Der Zeitraum der Analyse bezieht sich auf die Jahre 2005 bis 2007, da erst seit 2005 durchgängig Informationen über die Anzahl der Übernahmen in unbefristete Arbeitsverträge vorliegen. Es werden nur Betriebe berücksichtigt, die im ersten Halbjahr des jeweiligen Jahres befristet Beschäftigte eingesetzt, übernommen oder entlassen haben. Betriebe ohne befristet Beschäftigte bleiben außen vor, da diese keine Übernahmen durchführen können. Ausgangspunkt der empirischen Modellierung ist die Anzahl der Über- 
nahmen in den Betrieben. Da diese abhängige Variable nichtnegativ und ganzzahlig ist, bieten sich Zähldatenmodelle an (Hilbe 2007). Das Basismodell ist die Poisson-Regression.

Treten in der abhängigen Variable, hier also in der Anzahl der Übernahmen, zu viele Nullen auf, liefert eine Poisson-Regression verzerrte Ergebnisse (Hardin / Hilbe 2007). Abhilfe schafft eine sogenannte Zero-Inflated-Poisson-Regression (ZIP) (Lambert 1992). Die Nullen, die in dem sogenannten ,Inflationsteil' modelliert werden, können sowohl einer binomialen (systematische Nullen) als auch einer Poissonverteilung (unsystematische Nullen) entsprungen sein. Beide Verteilungen werden simultan als datengenerierende Prozesse unterstellt. Unsystematisch heißt, dass sich die Betriebe ohne Übernahmen nicht von jenen mit Übernahmen unterscheiden, so dass die Nullen als zufällig angesehen werden können. Ob die Anzahl der Nullen in den vorliegenden Daten zu hoch ist, kann auf der Grundlage eines Vuong Tests entschieden werden (Greene 1994). Liegt schließlich in einem Zero-Inflated-Poisson-Modell Überdisper$\operatorname{sion}^{13}$ vor, sind die Koeffizienten inkonsistent, da die Likelihood-Schätzung explizit auf der Poisson-Wahrscheinlichkeit für das Auftreten einer Null basiert (Ridout et al. 2001). In diesem Fall wird auf eine Zero-Inflated-Negative-Binomial-Regression (ZINB) zurückgegriffen. Der Unterschied zum ZIP-Modell besteht in der flexibleren Varianzannahme.

Um Aussagen über die betrieblichen Bestimmungsgrößen der Übernahme befristet Beschäftigter zu treffen, könnten alternativ direkt Übernahmequoten modelliert werden. ${ }^{14}$ Die Übernahmequote bestimmt sich aus $Q_{\ddot{U}}=Y / N$. Y ist die Anzahl der Übernahmen in unbefristete Beschäftigung und $\mathrm{N}$ ist die Summe aus den Übernahmen und der Anzahl der beendeten befristeten Beschäftigungsverhältnisse. Das Problem einer direkten Betrachtung der Quote ist allerdings, dass dann sowohl die Variation des Zählers als auch des Nenners den Wert der abhängigen Variable beeinflusst und die Interpretation der Ergebnisse erschwert wird. Um dies zu vermeiden, wird hier lediglich die Anzahl der Übernahmen auf einen Vektor unabhängiger Variablen regressiert. Damit die Ergebnisse dennoch als Quote interpretierbar sind und einen größenspezifischen Bezugspunkt haben, wird der logarithmierte Nenner (also die logarithmierte Anzahl aller Abgänge aus befristeter Beschäftigung) als zusätzlicher Regressor in der Schätzung berücksichtigt, sein Koeffizient jedoch auf eins restringiert. Dies lässt sich für die Poisson- und die Negative-Binomial-Regression ersehen aus

$$
\text { (1) } y_{i t}=e^{\left(x_{i t}^{\prime} \beta+\ln \left(N_{i, t}\right)\right.}=e^{\left(x_{i, t}^{\prime} \beta\right)} N_{i t} \text {, }
$$

so dass

$$
\text { (1), } \frac{y_{i t}}{N_{i t}}=e^{\left(x^{\prime}{ }_{i t} \beta\right)}
$$

gilt. Dabei ist $y_{i t}$ die Anzahl der Übernahmen, $N_{i t}$ die Summe aus den Übernahmen und der Anzahl der beendeten befristeten Beschäftigungsverhältnisse und $x_{i t}$ ein Vektor exogener Variablen für Unternehmen i zum Zeitpunkt t. $\beta$ ist der Koeffizientenvektor aus der Likelihood-

13 Die Varianzannahme, die in diesem Zusammenhang getroffen wird, ist allerdings für die meisten Daten inadäquat, da die Varianz i. d. R. den Erwartungswert der abhängigen Variablen übersteigt (Maddala 1983). Man spricht in diesem Fall von Überdispersion. Dies ist für die Koeffizienten zunächst kein Problem (Gourieroux et al. 1984), die errechneten Signifikanzen sind hingegen unzuverlässig. Eine Möglichkeit damit umzugehen besteht darin, die Standardfehler zu skalieren oder per Bootstrapping zu bestimmen (Hilbe 2007).

14 Alternativ wurde ein Logit-Modell für Anteilswerte nach Papke/Wooldridge (1996) geschätzt. Die Ergebnisse sind weitgehend identisch. Allerdings ist die Modellanpassung - gemessen durch ein verallgemeinertes $\mathrm{R}^{2}$-(Hardin/Hilbe 2003) des Zähldatenmodells besser. 
Schätzung. Im Rahmen der Zero-Inflated-Modelle ist der rechte Teil von (1) mit der Wahrscheinlichkeit zu multiplizieren, dass $y_{i t}$ größer Null ist. Die Schätzung erfolgt gepoolt über die drei Wellen 2005 bis $2007 .{ }^{15}$ Um eine daraus resultierende Unterschätzung der Standardfehler zu vermeiden, werden diese im Hinblick auf einen eindeutigen Betriebsidentifikator geclustert (Rogers 1993).

Folgende Variablen gehen in das Modell ein: Zunächst werden Informationen über die erwarteten Personalprobleme der Betriebe in die Schätzung einbezogen. ${ }^{16}$ Zur Abbildung des Rekrutierungsproblems werden die Variablen „Personalmangel“ und „Schwierigkeiten bei der Rekrutierung von Fachkräften“ berücksichtigt. Das Diskontinuitätsproblem wird mit den Problemen „zu hoher Personalstand“ und „hohe Personalfluktuation“ erfasst, wobei ersteres gegen, letzteres für Übernahmen spricht. Um die Bedeutung befristeter Verträge abzubilden, wird der Anteil befristet Beschäftigter an der betrieblichen Gesamtbeschäftigung in das Modell aufgenommen. Ein hoher Anteil an Befristungen deutet darauf hin, dass Befristungen nicht im Sinne eines Screening-Instruments genutzt werden, sondern als flexible Ergänzung der Stammbelegschaft. Es wird daher ein negativer Zusammenhang zwischen dem Befristungsanteil und der Übernahmequote erwartet.

Zwei Dummies bilden die erwartete Entwicklung des Geschäftsvolumens ab: Steigendes und sinkendes Geschäftsvolumen (Referenz: unverändertes Geschäftsvolumen und Unsicherheit über Entwicklung). Bei Betrieben, die einen Anstieg ihres Geschäftsvolumens erwarten, wird auch eine höhere Übernahmequote vermutet, da in Zukunft ebenso mit einem höheren Personalbedarf zu rechnen ist. Umgekehrtes gilt für einen erwarteten Rückgang der Entwicklung des Geschäftsvolumens. Die Beschäftigungsentwicklung wird mit der Wachstumsrate der sozialversicherungspflichtigen Beschäftigung im Vergleich zum Vorjahr abgebildet. ${ }^{17}$ Es ist anzunehmen, dass in wachsenden Betrieben Befristungen eher dem Beschäftigungsaufbau mit verlängerter Probezeit dienen. Übernahmen sind demnach in wachsenden Betrieben wahrscheinlicher. Darüber hinaus wird berücksichtigt, ob die Betriebe ins Ausland exportieren. Exportorientierte Betriebe sind einem erhöhten internationalen Wettbewerb ausgesetzt, was sich in einem erhöhten Flexibilitätsbedarf der Betriebe und geringer Übernahmebereitschaft ausdrücken könnte, sofern nicht auf alternative Flexibilisierungsstrategien zurückgegriffen wird.

Ein weiterer Indikator für die Lösungsstrategie des Diskontinuitätsproblems ist der Personalumschlag des Betriebs (vgl. Hohendanner / Janik 2008). Ein Maß hierfür ist die so genannte Churning-Rate ${ }^{18}$ (CR), die den Teil der Arbeitskräftemobilität beschreibt, der sich nicht aus der Veränderung der Anzahl der Mitarbeiter im Betrieb erklärt. Ein negativer Zusammenhang zwischen Churning-Rate und Übernahmequote befristeter Beschäftigung deutet darauf hin, dass Befristungen im Sinne der These des extrempoligen Kündigungsschutzes als arbeits-

15 Die Modelle ermöglichen somit partielle Korrelationsanalysen, kausale Aussagen werden nicht vorgenommen.

16 Die Betriebe wurden im Jahr 2004 und 2006 zu den Personalproblemen befragt, die sie in den nächsten zwei Jahren erwarten. Die Angaben der Betriebe aus dem Jahr 2004 wurden daher auf das Jahr 2005 und 2006, die Angaben von 2006 auf das Jahr 2007 übertragen.

17 Die Wachstumsrate basiert auf der Formel $2 *\left(\mathrm{~N}_{\mathrm{t}}-\mathrm{N}_{\mathrm{t}-1}\right) /\left(\mathrm{N}_{\mathrm{t}}+\mathrm{N}_{\mathrm{t}-1}\right)$, wobei $\mathrm{N}$ die Anzahl der Beschäftigten in einer Beschäftigungsform bezeichnet und t das aktuelle Jahr. Die Wachstumsrate ist zwischen -2 und 2 restringiert, um den Auf- und Abbau sozialversicherungspflichtiger Beschäftigung symmetrisch abzubilden und für Ausreißer zu kontrollieren. Die einfache Wachstumsrate $\left(\mathrm{N}_{\mathrm{t}}-\mathrm{N}_{\mathrm{t}-1}\right)$ / $\mathrm{N}_{\mathrm{t}-1}$ kann bei negativem Wachstum keine Werte unter -1 annehmen, im positiven Bereich besteht jedoch keine Begrenzung.

18 Sie wird wie folgt berechnet: $C R=(H+S-|H-S|) / L$, wobei $\mathrm{H}$ für die Zahl der Einstellungen, $\mathrm{S}$ für die Zahl der Abgänge und L für die durchschnittliche Anzahl der Beschäftigten im Beobachtungszeitraum steht. 
rechtliche Ausweichstrategie genutzt werden. Die nachgefragte Arbeitsmenge bleibt zwar konstant, Übergänge in dauerhafte Beschäftigungsverhältnisse werden aber vermieden, um Handlungsspielräume zu erhalten.

Eine weitere Variable, die diesen Zusammenhang abbildet, ist der Anteil der Arbeitgeberkündigungen an allen Abgängen aus dem Betrieb. Ein niedriger Anteil ist ein Hinweis auf einen hohen betrieblichen Bestandsschutz der regulär Beschäftigten. Ein negativer Zusammenhang zur Übernahmequote ist ein Hinweis auf Befristungen als Flexibilitätspuffer und Umgehungsinstrument des Kündigungsschutzes. Des Weiteren wird ein Dummy für die Existenz eines Betriebsrates sowie das Vorhandensein eines Tarifvertrags im Modell berücksichtigt, da dort ein stärkerer Bestandsschutz für die Stammbelegschaft - etwa im öffentlichen Dienst - verankert sein kann.

Wirtschaftliche Schwankungen werden insbesondere im verarbeitenden Gewerbe über den Einsatz von Leiharbeitern abgewickelt (Bellmann et al. 2009). Betriebe mit einem hohen Leiharbeiteranteil verwenden daher - so die These - Befristungen eher als Screeninginstrument. Die Flexibilität wird über Leiharbeitsfirmen abgesichert. Geht ein hoher Leiharbeiteranteil hingegen mit geringen Übernahmequoten befristeter Verträge einher, würde dies für eine komplementäre Nutzung von Befristungen und Leiharbeit als Flexibilitätspuffer sprechen (Pfeifer 2005). ${ }^{19}$ Die Verfügbarkeit von Arbeitskräften wird mit dem Anteil der offenen Stellen an der Gesamtbeschäftigung und dem Anteil der Arbeitnehmerkündigungen an allen Abgängen im Betrieb modelliert. Je höher der Anteil der offenen Stellen und der Arbeitnehmerkündigungen, umso größer ist das Rekrutierungsproblem des Betriebs und umso wahrscheinlicher sind Übernahmen in unbefristete Verträge. Fehlt den Betrieben die Möglichkeit der Entfristung - etwa aufgrund fehlender Personalmittel in sozialen Einrichtungen - können befristete Arbeitnehmer durch Kündigungen dem Auslaufen ihrer befristeten Verträge zuvorkommen. Dies ist jedoch davon abhängig, ob sie über entsprechende ,Exitoptionen“ verfügen. Ist dies der Fall, wäre ein umgekehrter Zusammenhang zu erwarten.

Als weitere Variable wird der Durchschnittslohn in Vollzeitäquivalenten aufgenommen. Bei hohen Lohnkosten können Befristungen einerseits als Screeninginstrument fungieren. Gerade bei hohen Gehaltszahlungen möchte der Arbeitgeber sich über eine erweiterte Probezeit ein Bild von der Produktivität des Arbeitnehmers machen. Andererseits können hohe Lohnkosten auf senioritätsbasierte Lohnentwicklungen in geschlossenen betrieblichen Beschäftigungssystemen hindeuten. In diesen Fällen wird ein negativer Zusammenhang zwischen Übernahmequote und Lohnsumme erwartet, da Befristungen genutzt werden, um die Lohnkostenentwicklung zu begrenzen.

Als Indikator für die Betriebsgröße wird die Anzahl der Beschäftigten im Betrieb (logarithmiert) aufgenommen, da davon auszugehen ist, dass sich die Probleme betrieblicher Personalpolitik betriebsgrößenspezifisch unterscheiden. Zusätzlich wird ein Indikator berücksichtigt, der die Gültigkeit des allgemeinen Kündigungsschutzes im Betrieb approximiert. ${ }^{20}$ Zudem

19 In weiteren Spezifikationen wurden der Anteil geringfügig Beschäftigter, der Anteil Teilzeitbeschäftigter, der Anteil Auszubildender sowie Aus- und Eingliederungen als alternative Flexibilisierungsinstrumente berücksichtigt. Da die Variablen allesamt insignifikant sind, wurden sie in der Endspezifikation nicht aufgenommen.

20 Der Dummy hat den Wert 1, wenn im Betrieb 10 und mehr Beschäftigte tätig sind. Da der arbeitsrechtliche Schwellenwert des Kündigungsschutzgesetzes im IAB-Betriebspanel nicht exakt abgebildet werden kann, ist diese Dummyvariable lediglich ein Proxy. Des Weiteren wurde ein Modell geschätzt nur mit Betrieben ab 10 Beschäftigten. Damit werden nur Betriebe betrachtet, in denen der allgemeine Kündigungsschutz und das Betriebsverfassungsgesetz gilt. Die Ergebnisse unterscheiden sich allerdings nicht. 
werden Branchendummies aufgenommen. Als Referenzbranche fungiert das produzierende Gewerbe.

\section{Ergebnisse}

Nach den hochgerechneten Angaben des IAB-Betriebspanels 2007 nehmen Befristungen mit etwa sieben Prozent (Tabelle 2, erste Spalte) den ,dritten Rang' in der Reihe der atypischen Beschäftigungsformen nach Teilzeitarbeit und geringfügiger Beschäftigung ein, spielen gleichwohl eine weitaus größere Rolle als freie Mitarbeit, Leiharbeit oder Praktika (Bellmann et al. 2009). Über 80 Prozent der Betriebe in Deutschland haben weniger als zehn Beschäftigte (und fallen damit nicht unter den allgemeinen Kündigungsschutz). Nur jeder zehnte dieser Kleinbetriebe nutzt Befristungen. Bei Großbetrieben ist es genau umgekehrt. Nur einer von zehn Großbetrieben ab 250 Beschäftigten nutzt keine Befristungen (Tabelle 2). Aufgrund der deutschen Betriebsgrößenstruktur haben daher 83 Prozent aller Betriebe im Jahr 2007 keine befristeten Arbeitskräfte. ${ }^{21}$

Tabelle 2: Befristete Arbeitsverhältnisse in Deutschland in Jahr 2007 (Aggregierte Anteile in Prozent)

\begin{tabular}{|l|c|c|c|c|}
\hline & Einstellungen & Übernahmen & $\begin{array}{c}\text { Befristungs- } \\
\text { anteil }\end{array}$ & $\begin{array}{c}\text { Betriebe mit } \\
\text { Befristungen }\end{array}$ \\
\hline Land- und Forstwirtschaft & 76 & $\begin{array}{c}\text { geringe } \\
\text { Fallzahlen }\end{array}$ & 10 & 17 \\
\hline Produzierendes Gewerbe & 40 & 68 & 4 & 15 \\
\hline Produktionsbezogene Dienstleistungen & 45 & 41 & 9 & 13 \\
\hline Distributive Dienstleistungen & 33 & 63 & 5 & 15 \\
\hline Personenbezogene Dienstleistungen & 40 & 48 & 9 & 16 \\
\hline Soziale Dienstleistungen & 64 & 39 & 10 & 27 \\
\hline Betriebe in öffentlichem Eigentum & 70 & 25 & 11 & 46 \\
\hline gemeinnützige Betriebe & 76 & 31 & 16 & 45 \\
\hline Exportierende Betriebe & 44 & 62 & 5 & 24 \\
\hline 1 bis 10 Beschäftigte (svB) & 26 & 44 & 4 & 10 \\
\hline 11 bis 49 Beschäftigte (svB) & 40 & 66 & 6 & 39 \\
\hline 50 bis 249 Beschäftigte (svB) & 54 & 47 & 9 & 72 \\
\hline 250 und mehr Beschäftigte & 63 & 42 & 9 & 91 \\
\hline Westdeutschland & 44 & 51 & 7 & 18 \\
\hline Ostdeutschland & 49 & 40 & 9 & 16 \\
\hline Insgesamt & 45 & 48 & 7 & 17 \\
\hline
\end{tabular}

Einstellungen: Aggregierte Anteile befristeter Neueinstellungen an allen Neueinstellungen im 1. Halbjahr 2007

Übernahmen: Aggregierte Anteile der Übernahmen an allen Abgängen (Übernahmen und Beendigung) befristeter Beschäftigung im 1. Halbjahr 2006/2007

Befristungsanteil: Aggregierte Anteile befristet Beschäftigter an der betrieblichen Gesamtbeschäftigung am 30.6.2007

Betriebe mit Befristungen: Anteil der Betriebe mit mindestens einem befristet Beschäftigten an allen Betrieben am 30.6.2007

Quelle: IAB-Betriebspanel, hochgerechnete Angaben

21 Da jedoch die größeren Betriebe einen Großteil der deutschen Gesamtbeschäftigung stellen, sind lediglich 40 Prozent der Beschäftigten in Betrieben tätig, in denen keine befristeten Verträge eingesetzt werden. 
Die Bestandsgrößen erwecken damit zunächst den Eindruck, dass Befristungen kein Massenphänomen darstellen. Bei Betrachtung der aggregierten Anteile der Neueinstellungen auf Basis eines befristeten Vertrages an allen Neueinstellungen (Tabelle 2, zweite Spalte) wird allerdings deutlich, dass sie in der betrieblichen Personalpolitik quantitativ eine weitaus größere Rolle spielen: In den sozialen Dienstleistungen erfolgen 63 Prozent und in Betrieben in öffentlichem Eigentum 70 Prozent aller Neueinstellungen auf Basis eines befristeten Vertrages und können daher als Normaleinstellungsverhältnis gelten. Übernahmen sind hingegen vergleichsweise selten. In Betrieben in öffentlichem Eigentum beträgt die Übernahmequote nur 25 Prozent, in Einrichtungen des sozialen Dienstleistungssektors 40 Prozent. Ein ähnlicher Zusammenhang lässt sich bei größeren Betrieben ab 250 Beschäftigten erkennen. Umgekehrt ist es im produzierenden Gewerbe und den distributiven Dienstleistungen: Unterdurchschnittliche Befristungsquoten von vier und fünf Prozent, Einstellungsquoten von 40 und 33 Prozent stehen dort Übernahmequoten von 68 und 63 Prozent gegenüber. Ein ähnliches Muster ist in exportierenden Betrieben zu beobachten. In den deskriptiven Befunden finden sich somit keine Hinweise auf einen direkten Zusammenhang zwischen internationalem Wettbewerb und einem verstärkten Einsatz von befristeten Verträgen als Flexibilitätspuffer. Auch Leiharbeit, die zu 70 Prozent im Produktionssektor eingesetzt wird, trägt mit einem Beschäftigtenanteil von vier Prozent in diesem Sektor nur in geringem Ausmaß zur Lösung des Diskontinuitätsproblems (und zum Bedeutungsverlust der Normalarbeit) bei (Bellmann et al. 2008).

In Tabelle 3 sind die Ergebnisse des ZINB-Modells sowie die Teststatistiken der Vuong(Greene 1994) und Likelihood-Ratio-Tests (Hilbe 2007) abgetragen. ${ }^{22}$ Die erste Ergebnisspalte zeigt die Regressionsergebnisse des Zähldatenteils, die zweite Spalte zeigt die Ergebnisse des Inflation-Teils. Da das Inflationsmodell das Auftreten von Nullen (und nicht etwa von Einsen wie etwa im Logitmodell) modelliert, müssen die Vorzeichen umgekehrt gelesen werden.

Tabelle 3: Zero-Inflated Negativ-Binomial Modell: Determinanten der Übernahme befristet Beschäftigter

\begin{tabular}{|l|c|c|}
\hline & $\begin{array}{c}\text { Anzahl der } \\
\text { Übernahmen }\end{array}$ & $\begin{array}{c}\text { Inflationsmo- } \\
\text { dell }\end{array}$ \\
\hline Betrieb erwartet Problem eines zu hohen Personalstand & $-0.086^{* * *}$ & $0.669^{* * *}$ \\
\hline & $(2.83)$ & $(4.53)$ \\
\hline Betrieb erwartet Problem hoher Personalfluktuation & 0.034 & $0.383^{*}$ \\
\hline & $(0.62)$ & $(1.67)$ \\
\hline Betrieb erwartet Personalmangel & $0.109^{* * *}$ & -0.310 \\
\hline & $(2.67)$ & $(1.03)$ \\
\hline Betrieb erwartet Schwierigkeiten, Fachkräfte zu rekrutieren & -0.029 & $-0.556^{* * *}$ \\
\hline & $(1.30)$ & $(3.50)$ \\
\hline Anteil Arbeitgeberkündigungen an allen Abgängen & $0.465^{* * *}$ & $-1.859^{* * *}$ \\
\hline & $(9.94)$ & $(5.57)$ \\
\hline Anteil Arbeitnehmerkündigungen an allen Abgängen & $0.462^{* * *}$ & $-1.882^{* * *}$ \\
\hline & $(15.99)$ & $(8.05)$ \\
\hline Anteil offener Stellen an der Gesamtbeschäftigung & $0.761 *$ & -0.255 \\
\hline & $(1.85)$ & $(0.38)$ \\
\hline
\end{tabular}

22 Überdies ergibt ein Wald-Test, dass es zulässig ist, den Koeffizienten für die Anzahl der Abgänge aus Befristungen auf eins zu restringieren. 


\begin{tabular}{|c|c|c|}
\hline & $\begin{array}{c}\text { Anzahl der } \\
\text { Übernahmen }\end{array}$ & $\begin{array}{l}\text { Inflationsmo- } \\
\text { dell }\end{array}$ \\
\hline Wachstumsrate sozialversicherungspflichtiger Beschäftigung & $0.413 * * *$ & $-1.414 * * *$ \\
\hline (um ein Jahr verzögert) & $(4.74)$ & $(4.40)$ \\
\hline \multirow[t]{2}{*}{ Churning Rate } & $-1.154 * * *$ & $1.696 * * *$ \\
\hline & $(6.03)$ & $(3.89)$ \\
\hline \multirow[t]{2}{*}{ Anteil Befristungen } & $-1.056^{* * *}$ & $2.113 * * *$ \\
\hline & $(7.39)$ & $(5.98)$ \\
\hline \multirow[t]{2}{*}{ Anteil Leiharbeiter } & $0.398 * * *$ & -1.299 \\
\hline & $(3.22)$ & $(0.92)$ \\
\hline \multirow[t]{2}{*}{ Erwartung eines Anstiegs des Geschäftsvolumens } & $0.078 * * *$ & $-0.721 * * *$ \\
\hline & $(3.48)$ & $(4.51)$ \\
\hline \multirow[t]{2}{*}{ Erwartung eines Absinkens des Geschäftsvolumens } & $-0.165 * * *$ & $0.378 * *$ \\
\hline & $(4.54)$ & $(2.42)$ \\
\hline \multirow[t]{2}{*}{ Betrieb hat ins Ausland exportiert } & 0.006 & 0.003 \\
\hline & $(0.21)$ & $(0.01)$ \\
\hline \multirow[t]{2}{*}{ Betrieb ist mehrheitlich in öffentlichem Eigentum } & $-0.197 * * *$ & $0.293 *$ \\
\hline & $(4.47)$ & $(1.72)$ \\
\hline \multirow{2}{*}{ Durchschnittslohn in Vollzeitäquivalenten (Log.) } & $-0.122 * * *$ & $-0.306^{*}$ \\
\hline & $(3.75)$ & $(1.93)$ \\
\hline \multirow[t]{2}{*}{ Tarifvertrag } & 0.025 & -0.069 \\
\hline & $(1.09)$ & $(0.44)$ \\
\hline \multirow[t]{2}{*}{ Betriebs-, Personalrat oder Mitarbeitervertretung } & -0.037 & -0.190 \\
\hline & $(1.21)$ & $(1.01)$ \\
\hline \multirow{2}{*}{ Gültigkeit des allgemeinen Kündigungsschutzes (Proxy) } & $-0.142 * * *$ & -0.605 \\
\hline & $(2.76)$ & $(1.07)$ \\
\hline \multirow[t]{2}{*}{ Gesamtbeschäftigung (log.) } & $-0.062 * * *$ & $0.336 * * *$ \\
\hline & $(5.28)$ & $(6.82)$ \\
\hline \multirow[t]{2}{*}{ West- und Ostdeutschland } & -0.046 & $0.457 * * *$ \\
\hline & $(1.62)$ & $(3.37)$ \\
\hline \multirow{2}{*}{ Land- und Forstwirtschaft } & -0.147 & -0.958 \\
\hline & $(1.29)$ & $(1.51)$ \\
\hline \multirow[t]{2}{*}{ Produktionsbezogene Dienstleistungen } & 0.048 & 0.009 \\
\hline & $(1.26)$ & $(0.04)$ \\
\hline \multirow[t]{2}{*}{ Distributive Dienstleistungen } & 0.009 & $-0.478^{*}$ \\
\hline & $(0.26)$ & $(1.69)$ \\
\hline \multirow[t]{2}{*}{ Personenbezogene Dienstleistungen } & 0.005 & 0.102 \\
\hline & $(0.07)$ & $(0.33)$ \\
\hline \multirow[t]{2}{*}{ Soziale Dienstleistungen (inklusive öffentlicher Dienst) } & $-0.114 * * *$ & -0.146 \\
\hline & $(3.34)$ & $(0.71)$ \\
\hline \multirow[t]{2}{*}{2006} & 0.008 & $-0.259 * *$ \\
\hline & $(0.35)$ & $(1.98)$ \\
\hline
\end{tabular}




\begin{tabular}{|l|c|c|}
\hline & $\begin{array}{c}\text { Anzahl der } \\
\text { Übernahmen }\end{array}$ & $\begin{array}{c}\text { Inflationsmo- } \\
\text { dell }\end{array}$ \\
\hline 2007 & $0.093^{* * *}$ & $-0.304^{* *}$ \\
\hline & $(4.33)$ & $(2.28)$ \\
\hline Konstante & $0.823^{* * *}$ & -1.068 \\
\hline \multicolumn{2}{|c|}{$(3.22)$} & $(0.85)$ \\
\hline Beobachtungen & 7741 & 7741 \\
\hline Wahl des Zähldatenmodells: (Vuong- und LR-Tests) & \multicolumn{2}{|c|}{$11.49^{* * *}$} \\
\hline ZIP > POISSON & \multicolumn{2}{|c|}{$8027.45^{* * *}$} \\
\hline NBREG > POISSON & \multicolumn{2}{|c|}{$13.80^{* * *}$} \\
\hline ZINB > NBREG & $4171,692^{* * *}$ \\
\hline ZINB > ZIP & \multicolumn{2}{|c|}{0.1928} \\
\hline Wald-Test: & \multicolumn{2}{|c|}{}
\end{tabular}

Da der Nenner der Übernahmequote (Anzahl der Abgänge aus befristeten Verträgen) in die Liste der unabhängigen Variablen aufgenommen wird und auf 1 restringiert werden soll, muss zunächst mittels eines Wald-Tests überprüft werden, ob der Koeffizient bei freier Schätzung signifikant von 1 abweicht. Der Wald-Test ist insignifikant. Daher kann die Variable auf 1 restringiert werden (vgl. Faraway 2006). Robuste z-Werte in Klammern, * signifikant auf $10 \%$; ** signifikant auf $5 \%$; *** signifikant auf $1 \%$; Gepooltes Modell nach 4890 Betrieben geclustert; Quelle: IAB-Betriebspanel 2005-2007

Werden Befristungen entsprechend der Diskontinuitätsthese als Flexibilitätspuffer genutzt, sind die Übernahmechancen gering. Hinweise hierfür bietet der negative Zusammenhang zwischen dem Personalproblem ,zu hoher Personalbestand“ und den Übernahmen. Weitere Anhaltspunkte liefert der negative Zusammenhang zwischen dem Anteil befristet Beschäftigter und der Übernahmequote. Die Flexibilitätspufferfunktion bestätigt sich auch anhand der Churningrate. In Betrieben mit hohem Personalaustausch ohne entsprechender Änderung der Gesamtzahl der Beschäftigten sind Übernahmen unwahrscheinlich. Dies spricht für ,,perverse Effekte“ eines extrempoligen Kündigungsschutzes. Betriebe vermeiden unbefristete Verträge obwohl sie einen kontinuierlichen Bedarf an Arbeitskräften haben. Der Bestandsschutz der Stammbelegschaft wird durch den Anteil der Arbeitgeberkündigungen modelliert. In Betrieben mit einem hohen Anteil an Arbeitgeberkündigungen sind Übernahmen wahrscheinlicher. Diese Betriebe sind in der Lage, ihre Anpassungsfähigkeit über Kündigungen aufrecht zu erhalten. Übernahmen sind daher mit geringeren Risiken behaftet. Die Übernahmechancen sinken zudem mit der Betriebsgröße. Dies ist ein Hinweis darauf, dass Befristungen gerade in Großbetrieben als Flexibilitätspuffer zum Schutz der Stammbelegschaft und zur Umgehung des Kündigungsschutzes eingesetzt werden.

Bei erwarteter positiver Geschäftsentwicklung sind Übernahmen wahrscheinlich, bei negativer unwahrscheinlich. Damit wird die Funktion von Befristungen als antizipierte Vermeidungsstrategie von Entlassungskosten untermauert. Befristete werden in unbefristete Verträge umgewandelt, wenn die ökonomischen Voraussetzungen gegeben sind. Dafür spricht auch der positive Zusammenhang zwischen dem Beschäftigungswachstum des Vorjahres und der Anzahl der Übernahmen. Ein hoher Leiharbeiteranteil geht mit einer höheren Übernahmequote befristet Beschäftigter einher. Die Befunde sprechen gegen die komplementäre Nutzung von Leiharbeit und Befristungen als Flexibilitätspuffer. Während Leiharbeit als Flexibilitätspuffer genutzt wird, fungieren Befristungen in diesen Betrieben eher als verlängerte Probezeit. Die Existenz eines Betriebsrats scheint die Übernahmepraxis der Betriebe nicht zu beeinflussen. Positive und negative Effekte können sich hierbei gegenseitig aufheben. Einerseits kann der Betriebsrat auf die Übernahme befristet Beschäftigter aktiv einwirken, andererseits erhöht sich 
über das Mitbestimmungsrecht des Betriebsrats der Bestandsschutz für unbefristet Beschäftigte, sodass Arbeitgeber mit der Erweiterung der Stammbelegschaft zögern.

Der negative Zusammenhang zwischen den Durchschnittslöhnen und der Anzahl der Übernahmen deutet einerseits darauf hin, dass Betriebe senioritätsbasierte Lohnsteigerungen bei Dauerbeschäftigung in geschlossenen betrieblichen Beschäftigungssystemen vermeiden wollen. Andererseits weist der positive Zusammenhang zwischen hohen Löhnen und der Tatsache, dass überhaupt übernommen wird (Inflationsteil des Modells), darauf hin, dass Betriebe ihre Beschäftigten über verlängerte Probezeiten besonders sorgfältig auswählen. Befristungen erfüllen demnach in Hochlohnbetrieben eine Screeningfunktion, wobei die Übernahmechancen relativ gering sind.

Wenn der Betrieb Schwierigkeiten hat, Personal zu rekrutieren oder zu halten, werden befristet Beschäftigte übernommen (Rekrutierungsthese). Dies zeigt sich vor allem an der positiven Korrelation zwischen dem Anteil der offenen Stellen, dem Anteil der Arbeitnehmerkündigungen sowie den Personalproblemen „Schwierigkeiten Fachkräfte zu finden“ und „Personalmangel“ und den Übernahmen. ${ }^{23}$ Die Bedeutung der Verhandlungspositionen der Vertragsparteien zeigt auch der negative Zusammenhang zwischen Befristungsanteil und Übernahmewahrscheinlichkeit: Wenn der Betrieb keine Schwierigkeiten hat, Arbeitsverträge auf Basis eines befristeten Vertrages zu schließen, deutet dies auf seine günstige Verhandlungsposition hin. Übernahmen sind für den Arbeitgeber dann nicht notwendig, da Befristungen oder ,Recalls‘ die günstigere Alternative darstellen. Damit zeigt sich auch, dass die Zunahme atypischer Beschäftigungsverhältnisse bzw. die Erosion von Normalarbeit eng mit der Verhandlungssituation der beteiligten Akteure auf dem Arbeitsmarkt verknüpft ist. Ein etwaiger Fachkräftemangel würde auf Basis der Ergebnisse bedeuten, dass die Anzahl an Übernahmen zunimmt und die Anzahl an Befristungen im Bereich der nachgefragten Fachkräfte zurückgeht.

Die branchenspezifischen Ergebnisse im Gesamtmodell (Tabelle 3) zeigen, dass die Übernahmechancen im sozialen Dienstleistungsbereich und Betrieben in öffentlichem Eigentum geringer sind als im produzierenden Gewerbe und in exportorientierten Betrieben. Besonders gut scheinen die Übernahmechancen in produktionsorientierten Dienstleistungsbranchen auszufallen. In Betrieben, die dem internationalen Wettbewerb ausgesetzt sind, stehen die Übernahmechancen nicht schlechter, obwohl davon auszugehen ist, dass sich das Diskontinuitätsproblem dort in verschärfter Weise stellt.

Um den unterschiedlichen personalpolitischen Strategien im produzierenden Gewerbe und den sozialen Dienstleistungen genauer auf den Grund zu gehen, werden in einem weiteren Schritt getrennte Modelle für die beiden Sektoren geschätzt (Tabelle 4). Aufschluss über die unterschiedliche Ausstattung hinsichtlich der untersuchten Einflussfaktoren der Sektoren geben die deskriptiven Befunde der getrennten Schätzungen (Tabelle 5). Die getrennten Analysen ermöglichen es, Unterschiede in den Verhaltensweisen und Reaktionen auf bestimmte personalpolitische Probleme zu identifizieren. Dazu können die Ergebnisse aus den beiden Schätzungen mithilfe eines generalisierten Hausman-Tests auf der Grundlage einer „SeeminglyUnrelated Cluster-Adjusted Sandwich-Estimators“ (Weesie 1999) verglichen werden. Da die Effekte der Variablen in einem ZINB-Modell auch von den anderen berücksichtigen Größen abhängen, werden nicht die Koeffizienten der beiden Schätzungen sondern die entsprechenden marginalen Effekte miteinander verglichen. ${ }^{24}$

23 Die positive Korrelation zwischen „Schwierigkeiten Fachkräfte zu rekrutieren“ und den Übernahmen zeigt sich lediglich im Inflationsteil des ZINB-Modells.

24 Die Berechnung der marginalen Effekte sowie der Test wurden mithilfe von STATA manuell vorgenommen. Eine ausführliche Darstellung der Vorgehensweise findet sich in Gerner/Stegmaier (2009). 
Tabelle 4: Determinanten der Übernahme befristet Beschäftigter. Getrennte Modelle für den sozialen Dienstleistungssektor und das produzierende Gewerbe. Marginale Effekte mit Test auf Unterschiede

\begin{tabular}{|c|c|c|c|}
\hline & $\begin{array}{l}\text { Produzierendes } \\
\text { Gewerbe }\end{array}$ & $\begin{array}{l}\text { Soziale Dienst- } \\
\text { leistungen }\end{array}$ & $\begin{array}{l}\chi^{2} \text {-Teststa- } \\
\text { tistik }\end{array}$ \\
\hline $\begin{array}{l}\text { Betrieb erwartet Problem eines } \\
\text { zu hohen Personalstands }\end{array}$ & $\begin{array}{c}-0,027 * * * \\
(-4,018)\end{array}$ & $\begin{array}{c}0,002 \\
(0,089)\end{array}$ & 1.65 \\
\hline $\begin{array}{l}\text { Betrieb erwartet Problem } \\
\text { hoher Personalfluktuation }\end{array}$ & $\begin{array}{c}0,020 \\
(0,863)\end{array}$ & $\begin{array}{c}0,029 \\
(0,653)\end{array}$ & 0.03 \\
\hline Betrieb erwartet Personalmangel & $\begin{array}{c}0,004 \\
(0,576)\end{array}$ & $\begin{array}{c}0,060 \\
(1,626)\end{array}$ & 2.18 \\
\hline $\begin{array}{l}\text { Betrieb erwartet Schwierigkeiten, } \\
\text { Fachkräfte zu rekrutieren }\end{array}$ & $\begin{array}{c}-0,002 \\
(-0,501)\end{array}$ & $\begin{array}{c}0,001 \\
(0,032)\end{array}$ & 0.02 \\
\hline $\begin{array}{l}\text { Anteil Arbeitgeberkündigungen } \\
\text { an allen Abgängen }\end{array}$ & $\begin{array}{c}0,061 * * * \\
(8,699)\end{array}$ & $\begin{array}{c}0,224 * * * \\
(5,831)\end{array}$ & $17.49 * * *$ \\
\hline $\begin{array}{l}\text { Anteil Arbeitnehmerkündigungen } \\
\text { an allen Abgängen }\end{array}$ & $\begin{array}{l}0,063 * * * \\
(12,049)\end{array}$ & $\begin{array}{l}0,257 * * * \\
(10,134)\end{array}$ & $56.41 * * *$ \\
\hline Anteil offener Stellen an der Gesamtbeschäftigung & $\begin{array}{c}0,086 \\
(0,872)\end{array}$ & $\begin{array}{l}-0,472 * * \\
(-2,072)\end{array}$ & $5.05 * *$ \\
\hline $\begin{array}{l}\text { Wachstumsrate sozialversicherungspflichtiger } \\
\text { Beschäftigung (aus dem Vorjahr) }\end{array}$ & $\begin{array}{c}0,067 * * * \\
(4,474)\end{array}$ & $\begin{array}{c}0,281^{* * *} \\
(2,379)\end{array}$ & $3.22 *$ \\
\hline Churning Rate & $\begin{array}{c}-0,493 * * * \\
(-7,190) \\
\end{array}$ & $\begin{array}{c}-1,699 * * * \\
(-8,148) \\
\end{array}$ & $30.22 * * *$ \\
\hline Anteil Befristungen & $\begin{array}{c}-0,051 \\
(-1,030)\end{array}$ & $\begin{array}{c}-0,334 * * * \\
(-3,416)\end{array}$ & $6.63 * * *$ \\
\hline Anteil Leiharbeiter & $\begin{array}{c}0,066 * * * \\
(2,977)\end{array}$ & $\begin{array}{l}-0,122^{*} \\
(-0,191)\end{array}$ & 0.09 \\
\hline Erwartung eines Anstiegs des Geschäftsvolumens & $\begin{array}{l}0,011^{* *} \\
(2,253) \\
\end{array}$ & $\begin{array}{c}0,054 * * * \\
(2,452)\end{array}$ & $3.68^{*}$ \\
\hline $\begin{array}{l}\text { Erwartung eines Absinkens } \\
\text { des Geschäftsvolumens }\end{array}$ & $\begin{array}{c}-0,030 * * * \\
(-4,701)\end{array}$ & $\begin{array}{c}-0,069 * * * \\
(-2,991)\end{array}$ & 2.56 \\
\hline Betrieb hat ins Ausland exportiert & $\begin{array}{c}0,001 \\
(0,283)\end{array}$ & $\begin{array}{c}-0,138 \\
(-1,178)\end{array}$ & 1.42 \\
\hline Betrieb ist mehrheitlich in öffentlichem Eigentum & $\begin{array}{c}-0,035^{* * *} \\
(-2,403)\end{array}$ & $\begin{array}{c}-0,061 * * * \\
(-3,391)\end{array}$ & 1.23 \\
\hline Durchschnittslohn in Vollzeitäquivalenten (log.) & $\begin{array}{c}-0,008 \\
(-0,898)\end{array}$ & $\begin{array}{c}0,018 \\
(0,552)\end{array}$ & 0.58 \\
\hline Tarifvertrag & $\begin{array}{c}-0,003 \\
(-0,707)\end{array}$ & $\begin{array}{c}-0,036 \\
(-1,870)\end{array}$ & $2.81 *$ \\
\hline Betriebs-, Personalrat oder Mitarbeitervertretung & $\begin{array}{l}-0,005 \\
(-1,057)\end{array}$ & $\begin{array}{c}-0,032 \\
(-0,923)\end{array}$ & 0.58 \\
\hline Gesamtbeschäftigung (log.) & $\begin{array}{c}-0,007 * * * \\
(-2,807)\end{array}$ & $\begin{array}{c}-0,044 * * * \\
(-4,931)\end{array}$ & $15.71 * * *$ \\
\hline
\end{tabular}




\begin{tabular}{|l|c|c|c|}
\hline & $\begin{array}{c}\text { Produzierendes } \\
\text { Gewerbe }\end{array}$ & $\begin{array}{c}\text { Soziale Dienst- } \\
\text { leistungen }\end{array}$ & $\begin{array}{c}\chi^{2} \text {-Teststa- } \\
\text { tistik }\end{array}$ \\
\hline Ostdeutschland & $-0,005$ & $-0,061^{* * *}$ & $8.53^{* * *}$ \\
$(-0,929)$ & $(-3,263)$ & \\
\hline 2006 & 0,003 & 0,023 & 1.02 \\
\hline 2007 & $(0,602)$ & $(1,182)$ & \\
\hline Beobachtungen & $0,014^{* * *}$ & $0,070^{* * *}$ & $6.30^{* * *}$ \\
& $(2,842)$ & $(3,190)$ & \\
\hline
\end{tabular}

Quelle: IAB-Betriebspanel 2005-2007, Robuste z-Werte in Klammern, * signifikant auf $10 \%$; ** signifikant auf $5 \%$; *** signifikant auf $1 \%$;

\section{Tabelle 5: Deskriptive Statistiken der ZINB-Modelle}

Grundsätzlich zeigen die Ergebnisse, dass Betriebe des sozialen Dienstleistungssektors einerseits anders auf personalpolitische Probleme reagieren (Tabelle 4), aber andererseits - und das zeigen die deskriptiven Statistiken in Tabelle 5 - unterscheiden sich die personalpolitischen Problemlagen zwischen den Sektoren deutlich. ${ }^{25}$

Die zentralen Ergebnisse seien an dieser Stelle herausgegriffen: Während der Anteil offener Stellen im Produktionssektor keinen Einfluss auf die Übernahme hat, zeigt sich im sozialen Dienstleistungssektor ein negativer Zusammenhang. Auch wenn offene Stellen vorhanden sind, werden befristet Beschäftigte nicht übernommen. Dies deutet darauf hin, dass den Betrieben die Möglichkeit der Entfristung fehlt - etwa aufgrund begrenzter Personalmittel. Die ungünstigere wirtschaftliche Situation in den sozialen Dienstleistungen zeigt sich dabei in den deskriptiven Mittelwerten der Erwartungen bezüglich des Geschäftsvolumens (Tabelle 5). Interessant ist hierbei, dass Betriebe des sozialen Dienstleistungssektors bei positiven Geschäftserwartungen stärker mit Übernahmen reagieren als im Produktionssektor (Tabelle 4). Würde sich die finanzielle Ausstattung der sozialen Dienste - was fraglich ist - verbessern, wäre ein vergleichsweise stärkerer Anstieg der Übernahmen zu erwarten. Einen ähnlichen Zusammenhang zeigt die Wachstumsrate der sozialversicherungspflichtigen Beschäftigung.

Der im Vergleich zum Produktionssektor geringere Mittelwert bei den Arbeitgeberkündigungen (Tabelle 5) zeigt die geringe Kündigungsneigung öffentlicher Arbeitgeber. Personalumschlag wird eben über Befristungen und nicht über reguläre Entlassungen realisiert. Dies zeigt auch der deskriptive Vergleich der Churningrate zwischen den Sektoren: Der Personalumschlag, der nicht zu einer Veränderung der Gesamtbeschäftigung führt, ist im sozialen Dienstleistungssektor doppelt so hoch ist wie im Produktionssektor. ${ }^{26}$ Ein zentrales Flexibilisierungsinstrument im Produktionssektor ist Leiharbeit. Im sozialen Dienstleistungssektor

25 Zusätzlich wurde eine Dekompositionsanalyse (vgl. Sinning et al. 2009) durchgeführt. Unter der Annahme, dass sich die Betriebe im Bereich der sozialen Dienstleistungen in Abhängigkeit der betrachteten Einflussgrößen ähnlich verhalten wie Betriebe des Produktionssektors, ließen sich 87 Prozent der Unterschiede im Übernahmeverhalten auf Unterschiede in den Einflussgrößen zurückführen. Dieses Ergebnis ist allerdings kaum aussagekräftig, da es massive Unterschiede im Verhalten der Betriebe in Abhängigkeit der Einflussgrößen zwischen den Sektoren gibt. Dies unterstreicht die Notwendigkeit der oben durchgeführten getrennten Analysen für die beiden Sektoren.

26 Zwischen Churningrate und Übernahmequote besteht sowohl im produzierenden Gewerbe als auch im sozialen Dienstleistungssektor ein negativer Zusammenhang (Tabelle 4). Dies zeigt, dass Betriebe in beiden Sektoren Befristungen durchaus als Flexibilitätspuffer nutzen. Allerdings ist die Effektstärke bei den sozialen Diensten wesentlich höher. 
spielt Leiharbeit hingegen praktisch keine Rolle. Der positive Zusammenhang zwischen Leiharbeiteranteil und Übernahmequote im Produktionssektor spricht gegen die komplementäre Nutzung von Leiharbeit und Befristungen als Flexibilitätsinstrument. Während Leiharbeit als Flexibilitätspuffer eingesetzt wird, fungieren Befristungen eher als Screeninginstrument.

Die getrennten Analysen veranschaulichen, dass die Einrichtungen des sozialen Dienstleistungssektors ihre Flexibilität vor allem über Befristungen sichern: Im öffentlichen Dienst verringert der hohe Bestandsschutz der Stammbelegschaft sowie die einseitig auf Befristungen, (Vor-)Ruhestandsregelungen und Versetzungen ausgerichtete Personalpolitik die Übernahmechancen (vgl. Bellmann et al. 2009). In den nicht-erwerbsorientierten und privaten sozialen Dienstleistungen spielen wiederum die quasi-marktliche Neuorganisation sowie die Abhängigkeit von zeitlich befristeten öffentlichen Mitteln eine Rolle. Im produzierenden Sektor werden Befristungen zwar auch zur Lösung des Diskontinuitätsproblems genutzt, aber in geringerem Ausmaß. Die Übernahmechancen fallen wesentlich günstiger aus als im Bereich der sozialen Dienstleistungen. Flexibilität wird im Produktionssektor eher über interne Flexibilität und Leiharbeit erzielt, wobei Leiharbeit quantitativ keine große Rolle spielt.

\section{Zusammenfassung und Fazit}

In vorliegender Studie wurde aufgezeigt, dass eine Zunahme befristeter Verträge kein unaufhaltsamer globaler Trend ist. Zwar ist ein Anstieg befristeter Neueinstellungen zu beobachten, am Ende einer Befristung steht allerdings häufig eine Übernahme in ein unbefristetes Arbeitsverhältnis. Im ersten Halbjahr 2007 hielten sich Übernahmen in unbefristete und das Auslaufen befristeter Verträge im selben Betrieb in etwa die Waage (Tabelle 2).

Die Übernahme in eine unbefristete Tätigkeit hängt dabei von der jeweiligen Funktion ab, die der befristete Vertrag im Zusammenhang mit der Lösung der dargestellten Probleme betrieblicher Beschäftigungsstrategien - Diskontinuität und Rekrutierung - erfüllt. Nutzen Betriebe Befristungen im Umgang mit betriebs- und branchenspezifischer Diskontinuität, ist die Chance auf Übernahme gering. Wenn für den Arbeitgeber - angesichts eines hohen Kündigungsschutzes - lediglich die Wahl zwischen (faktischer) Unkündbarkeit und dem Austausch eines befristet Beschäftigten besteht, stehen die Übernahmechancen vergleichsweise schlecht. Allerdings verbessern sich diese bei positiver wirtschaftlicher Entwicklung. Auch erhöhen sie sich, wenn die Verfügbarkeit gesuchter Fachkräfte eingeschränkt ist. Dieser Zusammenhang deutet auf die Bedeutung von Verhandlungsoptionen im Kontext der Befristungspraxis hin. Verfügen die Arbeitskräfte über ausreichende Optionen auf dem externen Arbeitsmarkt bei gleichzeitig fehlenden Alternativen für den Arbeitgeber, müssen Arbeitgeber dem in der Regel vorhandenen Wunsch nach einem unbefristeten Vertrag nachgeben.

Die Befristungs- und Übernahmepraxis ist branchenspezifisch äußerst unterschiedlich ausgeprägt. Einerseits zeigt sich, dass Befristungen insbesondere im öffentlichen Dienst und den sozialen Dienstleistungen vor allem als Flexibilitätsinstrument genutzt werden und Übernahmechancen dort begrenzt sind. Der inkrementelle Personalabbau, jährliche Haushaltspläne, der Kündigungsschutz im öffentlichen Dienst, die prekäre (öffentliche) Finanzierung und neue quasi-marktliche Organisationsformen in den sozialen Dienstleistungen spielen dabei eine wichtige Rolle. Andererseits wird deutlich, dass Betriebe des Produktionssektors sowie Betriebe, die dem internationalen Wettbewerb stark ausgesetzt sind, befristete Verträge nicht in besonderem Maße als Flexibilitätspuffer einsetzen. Der direkte Beitrag der ,Globalisierung im Sinne eines verschärften internationalen Wettbewerbs an der Erosion der Normalarbeit ist - zumindest was Befristungen betrifft - nicht deutlich erkennbar. In Betrieben des Produktionssektors gehen 2007 immerhin über zwei Drittel aller Abgänge aus Befristungen auf innerbetriebliche Übernahmen zurück (Tabelle 2). Auch zeigt sich, dass befristet Beschäftigte bei positiver Geschäftsentwicklung und ,Kündigungsfähigkeit‘ des Arbeitgebers übernommen 
werden. Insbesondere eine bessere bzw. nachhaltigere finanzielle Ausstattung von sozialen Diensten würde die Übernahmequoten möglicherweise erhöhen.

Arbeitsmarktpolitisch sind Befristungen ambivalent zu bewerten. Einerseits werden Befristungen zum Aufbau von Beschäftigung genutzt und erleichtern dem Arbeitgeber Einstellungen. Andererseits sind die Übernahmen seltener in Betrieben, die Befristungen als Flexibilitätspuffer nutzen. Insbesondere der hohe Anteil an befristeten Neueinstellungen bei gleichzeitig geringen Übernahmequoten im öffentlichen Dienst und den sozialen Dienstleistungsbranchen veranschaulichen, dass gerade in diesen Branchen die Gefahr einer Verstärkung sozialer Ungleichheiten besteht.

\section{Literatur}

Ashford, Susan J. / Cynthia Lee / Philip Bobko (1989): Content, causes, and consequences of job insecurity: a theory-based measure and substantive test, in: Academy of Management Journal 32, S. 803-829.

Auer, Peter (2007): Labour market security in between employment and social protection, Genf.

Bellmann, Lutz / Gabriele Fischer / Christian Hohendanner (2009): Betriebliche Dynamik und Flexibilität auf dem deutschen Arbeitsmarkt, in: Joachim Möller / Ulrich Walwei (Hrsg.), Handbuch Arbeitsmarkt 2009, IAB-Bibliothek 314, S. 359-401.

Blanchard, Oliver / Augustin Landier (2002): The Perverse Effects of Partial Labour Market Reforms: Fixed-Term Contracts in France, in: The Economic Journal 112, S. 214-244.

Blossfeld, Hans-Peter / Sandra Buchholz / Dirk Hofäcker / Heather Hofmeister / Karin Kurz / Melinda Mills (2008): Globalisierung und die Veränderung sozialer Ungleichheiten in modernen Gesellschaften. Eine Zusammenfassung der Ergebnisse des GLOBALIFE-Projektes, in: Kölner Zeitschrift für Soziologie und Sozialpsychologie 59, S.667-691.

Boockmann, Bernhard / Tobias Hagen (2002): Arbeitsplatzdynamik und befristete Verträge: Empirische Evidenz aus dem IAB-Betriebspanel für Baden-Württemberg, in: Mitteilungen aus der Arbeitsmarktund Berufsforschung 35, S. 385-396.

Boockmann, Bernhard / Tobias Hagen (2003): Works Councils and Fixed-Term Employment: Evidence from West German Establishments, in: Schmollers Jahrbuch 123, S. 359-381.

Boockmann, Bernhard / Tobias Hagen (2006): Befristete Beschäftigungsverhältnisse - Brücken in den Arbeitsmarkt oder Instrumente der Segmentierung?, Baden-Baden.

Büchtemann, Christoph F. (1990): „Deregulierung“ des Arbeitsmarktes: Begriffsbestimmung und sozialstaatliche Implikationen, in: Christoph F. Büchtemann / Helmut Neumann (Hrsg.), Mehr Arbeit durch weniger Recht? Chancen und Risiken der Arbeitsmarktflexibilisierung, Berlin, S. 229-244.

Capelli, Peter / David Neumark (2004): External Churning and Internal Flexibility: Evidence on the Functional Flexibility and Core-Periphery Hypotheses, in: Industrial Relations 43, S. 148-181.

Dahme, Heinz-Jürgen / Norbert Wohlfahrt (2006): Zur Entwicklungsdynamik sozialer Dienste im Wettbewerb. Ein Blick auf die Zukunft der Sozialen Arbeit, in: Sozial Extra 30, S. 28-32.

Dahrendorf, Ralf (1980): Im Entschwinden der Arbeitsgesellschaft. Wandlungen der sozialen Konstruktion des menschlichen Lebens, in: Merkur 34, S. 749-760.

Doeringer, Peter / Michael Piore (1971): Internal Labor Markets and Manpower Analysis, Lexington MA.

Dörre, Klaus (2006): Prekäre Arbeit. Unsichere Beschäftigungsverhältnisse und ihre sozialen Folgen, in: Arbeit 15, S. 181-193.

Faraway, Julian J. (2006): Extending the linear model with R., Boca Raton.

Fischer, Gabriele / Florian Janik / Dana Müller / Alexandra Schmucker (2009): The IAB Establishment Panel. Things users should know, in: Schmollers Jahrbuch 129, S.133-148. 
Gerner, Hans-Dieter / Jens Stegmaier (2009): Unsicherheit und betriebliche Weiterbildung. Eine empirische Analyse der Weiterbildungsaktivität unter Unsicherheit in KMU und Großbetrieben, in: Zeitschrift für Betriebswirtschaft, Special Issue 6, S.135-163.

Giddens, Anthony (1995): Die Konstitution der Gesellschaft, Frankfurt / Main, New York.

Giesecke, Johannes (2006): Arbeitsmarktflexibilisierung und soziale Ungleichheit, Wiesbaden.

Giesecke, Johannes / Martin Groß (2002): Befristete Beschäftigung: Chance oder Risiko?, in: Kölner Zeitschrift für Soziologie und Sozialpsychologie 54, S. 85-108.

Gourieroux, Christian / Alain Monfort / Alain Trognon (1984): Pseudo Maximum Likelihood Methods: Theory, in: Econometrica 52, S. 680-700.

Greene, William M. (1994): Accounting for excess zeros and sample selection in Poisson and negative binomial regression models, New York University Working Paper EC-94-10.

Gundert, Stefanie (2007): Befristete Beschäftigung bei Berufsanfängern und älteren Arbeitnehmern, Berlin.

Hagen, Tobias (2003): Does Fixed-Term Contract Employment Raise Firms' Adjustment Speed? Evidence from an Establishment Panel for West Germany, in: Jahrbücher für Nationalökonomie und Statistik 223, S. 403-421.

Hagen, Tobias / Bernhard Boockmann (2002): Determinanten der Nachfrage nach befristeten Verträgen, Leiharbeit und freier Mitarbeit: Empirische Analysen auf Basis des IAB-Betriebspanels, in: Lutz Bellmann / Arndt Kölling (Hrsg.), Betrieblicher Wandel und Fachkräftebedarf, Beiträge zur Arbeitsmarktund Berufsforschung 257, S. 198-235.

Hardin, James W. / Joseph M. Hilbe (2003): Generalized Estimating Equations, London.

Hardin, James W./ Joseph M. Hilbe (2007): Generalized Linear Models and Extensions, College Station, Texas.

Hellgren, Johnny / Sverke Magnus / Kerstin Isaksson (1999): A Two-Dimensional Approach to Job Insecurity: Consequences for Employee Attitudes and Well-Being, in: European Journal of Work and Organizational Psychology 8, S. 179-195.

Herrmann, Andrea (2008): Rethinking the link between labour market flexibility and corporate competitiveness: a critique of the institutionalist literature, in: Socio-Economic Review 6, S. 637-669.

Hilbe, Joseph M. (2007): Negative Binomial Regression, Cambridge.

Hohendanner, Christian / Lutz Bellmann (2007): Atypische Beschäftigung und betrieblicher Flexibilisierungsbedarf. Ergebnisse des IAB-Betriebspanels, in: Berndt Keller / Hartmut Seifert (Hrsg.), Atypische Beschäftigung. Flexibilisierung und soziale Risiken, Berlin, S. 27-43.

Hohendanner, Christian / Florian Janik (2008): Praktika und betriebliche Personalpolitik - Verbreitung und Nutzungsintensität von Praktika in deutschen Betrieben, in: Zeitschrift für Arbeitsmarktforschung 4, S. 471-487.

Jann, Werner/ Kai Wegrich / Jan Tiessen (2007): Bürokratisierung und Bürokratieabbau im internationalen Vergleich - wo steht Deutschland?, Berlin.

Kaiser, Ulrich / Friedhelm Pfeiffer (2001): Collective Wage Agreements and Firms' Employment Policies, LABOUR: Review of Labour Economics and Industrial Relations 10, S. 319-341.

Köhler, Christoph / Kai Loudovici / Olaf Struck (2007): Generalisierung von Beschäftigungsrisiken, in: Berliner Journal für Soziologie 17, S. 387-406.

Koller, Lena / Claus Schnabel / Joachim Wagner (2007): Schwellenwerte im Arbeitsrecht: Höhere Transparenz und Effizienz durch Vereinheitlichung, in: Perspektiven der Wirtschaftspolitik 8, S. 242-255.

Lambert, Diane (1992): Zero-inflated Poisson regression with an application to defets in manufacturing, in: Technometrics 34, S. 1-14.

Lengfeld, Holger / Tuuli-Marja Kleiner (2009): Flexible Beschäftigung und soziale Ungleichheit, in: Arbeit 18 , S. 46-62. 
Liebig, Stefan / Andrea Hense (2007): Die zeitweise Verlagerung von Arbeitskräften in die Arbeitslosigkeit: Eine ,neue“ personalpolitische Flexibilisierungsstrategie?, in: Zeitschrift für Arbeitsmarktforschung 4, S. 399-417.

Lutz, Burkhard (1987): Arbeitsmarktstruktur und betriebliche Arbeitskräftestrategie. Eine theoretischhistorische Skizze zur Entstehung betriebszentrierter Arbeitsmarktsegmentation, Frankfurt/ Main, New York.

Maddala, Gangadharrao S. (1983): Limited Dependent and Qualitative Variables in Econometrics, Cambridge.

McGinnity, Frances/ Antje Mertens / Stefanie Gundert (2005): A Bad Start? Fixed-Term Contracts and the Transition from Education to Work in West Germany, in: European Sociological Review 21, S. 359-374.

Meyer, Wolfgang / Christian Pfeifer (2005): Flexiblere Anpassung mit befristeten Beschäftigungsverhältnissen? Eine empirische Analyse mit Firmendaten für Niedersachsen, in: Lutz Bellmann / Olaf Hübler / Wolfgang Meyer / Gesine Stephan (Hrsg.), Institutionen, Löhne und Beschäftigung. Beiträge zur Arbeitsmarkt- und Berufsforschung 294, Nürnberg, S. 175-188.

Mückenberger, Ulrich (1985): Die Krise des Normalarbeitsverhältnisses - hat das Arbeitsrecht noch Zukunft?, in: Zeitschrift für Sozialreform 31, S. 415-475.

Nienhüser, Werner (2007): Betriebliche Beschäftigungsstrategien und atypische Arbeitsverhältnisse. Eine Erklärungsskizze aus Sicht einer politischen Personalökonomik, in: Berndt Keller / Hartmut Seifert (Hrsg.), Atypische Beschäftigung. Flexibilisierung und soziale Risiken, Berlin, S. 45-65.

OECD (2000): Employment Outlook 2000, Paris.

Offe, Claus (2008): Governance - „Empty signifier“ oder sozialwissenschaftliches Forschungsprogramm?, in: Gunnar F. Schuppert / Michael Zürn (Hrsg.), Governance in einer sich wandelnden Welt. Wiesbaden.

Papke, Leslie E. / Jeffrey M. Wooldridge (1996): Econometric methods for fractional response variables with an application to $401(\mathrm{~K})$ plan participation rates, in: Journal of Applied Econometrics 11, S. 619-632.

Parent-Thirion, Agnès / Enrique F. Macías / John Hurley / Greet Vermeylen (2007): Fourth European Working Conditions Survey, Dublin.

Pfeifer, Christian (2005): Flexibility, Dual Labour Markets, and Temporary Employment. Empirical Evidence from German Establishment Data, in: Management Revue 16, S. 404-422.

Pfeifer, Christian (2006): Warum beschäftigen Firmen befristete Arbeitnehmer und Leiharbeitskräfte? Eine theoretische und empirische Analyse mit Daten aus dem Hannoveraner Firmenpanel, in: Werner Nienhüser (Hrsg.), Beschäftigungspolitik von Unternehmen - Theoretische Erklärungsansätze und empirische Erkenntnisse, Mering, S. 197-224.

Ridout, Martin / Clarice G. B. Demétrio / John Hinde (2001): A score test for testing a zero-inflated poisson regression model against zero-inflated negative binomial alternatives, in: Biometrics, 57, S. 219-223.

Rifkin, Jeremy (1995): Das Ende der Arbeit und ihre Zukunft, Frankfurt / Main, New York.

Rogers, William H. (1993): Regression standard errors in clustered samples, in: Stata Technical Bulletin 13, S. 19-23.

Rudolph, Helmut (2005): Beschäftigungsformen: ein Maßstab für Flexibilität und Sicherheit, in: Martin Kronauer / Gudrun Linne (Hrsg.), Flexicurity. Die Bindung von Sicherheit an Flexibilität, Berlin, S. 97-126.

Sinning, Mathias / Markus Hahn / Thomas K. Bauer (2009): The Blinder-Oaxaca decomposition for nonlinear regression models, in: The Stata Journal 8, S. 480-492.

Struck, Olaf (2006): Flexibilität und Sicherheit. Empirische Befunde, theoretische Konzepte und institutionelle Gestaltung von Beschäftigungsstabilität, Wiesbaden. 
Vielle, Pascale; Walthery, Pierre (2003): Flexibility and social protection, Dublin.

Weesie, Jeroen (1999): Seemingly unrelated estimation and the cluster-adjusted estimator, in: Stata Technical Bulletin 52, S. 230-257.

Christian Hohendanner

Dr. Hans-Dieter Gerner

Institut für Arbeitsmarkt- und Berufsforschung

Der Bundesagentur für Arbeit

Regensburger Str. 104 90478 Nürnberg christian.hohendaner@iab.de hans-dieter.gerner@iab.de 


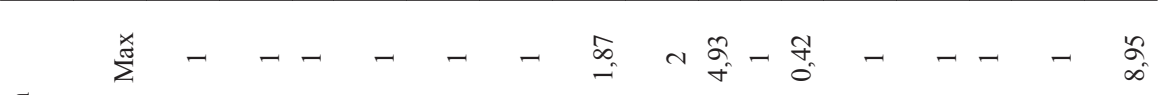

营总

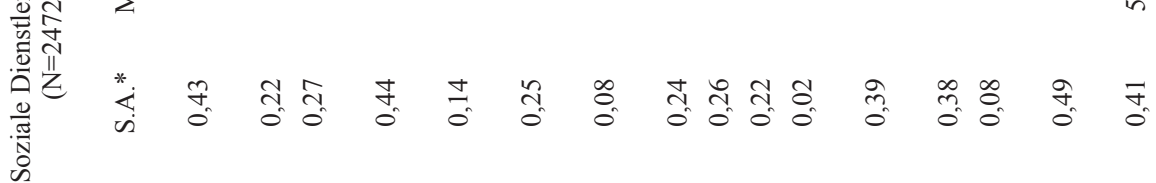

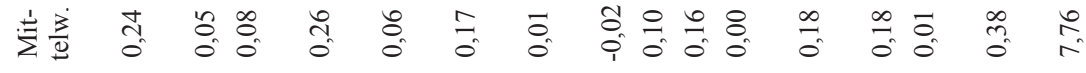

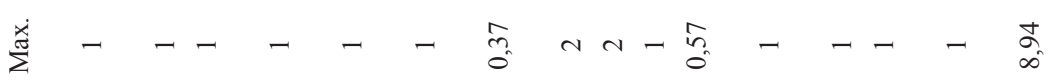
0
0
0
0
0
0
0
0
0
0
0
0
0
0
0

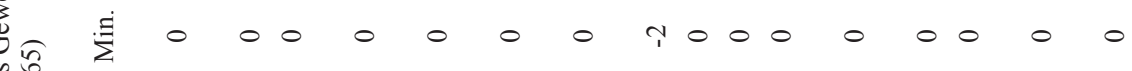

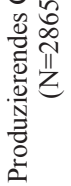

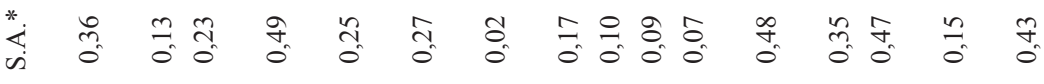

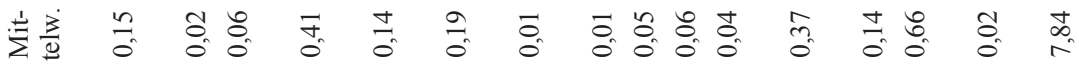

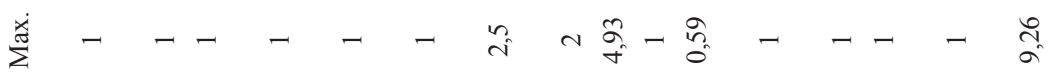

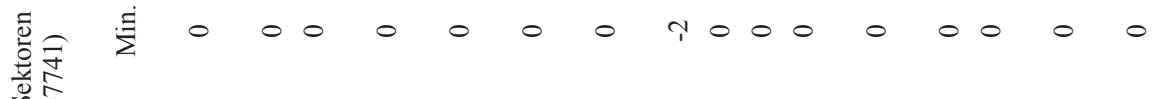

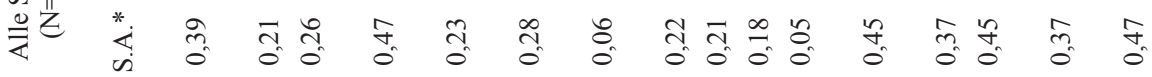

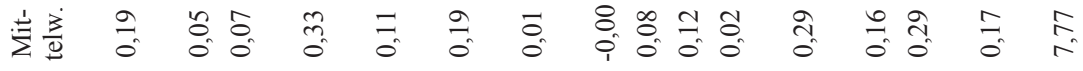

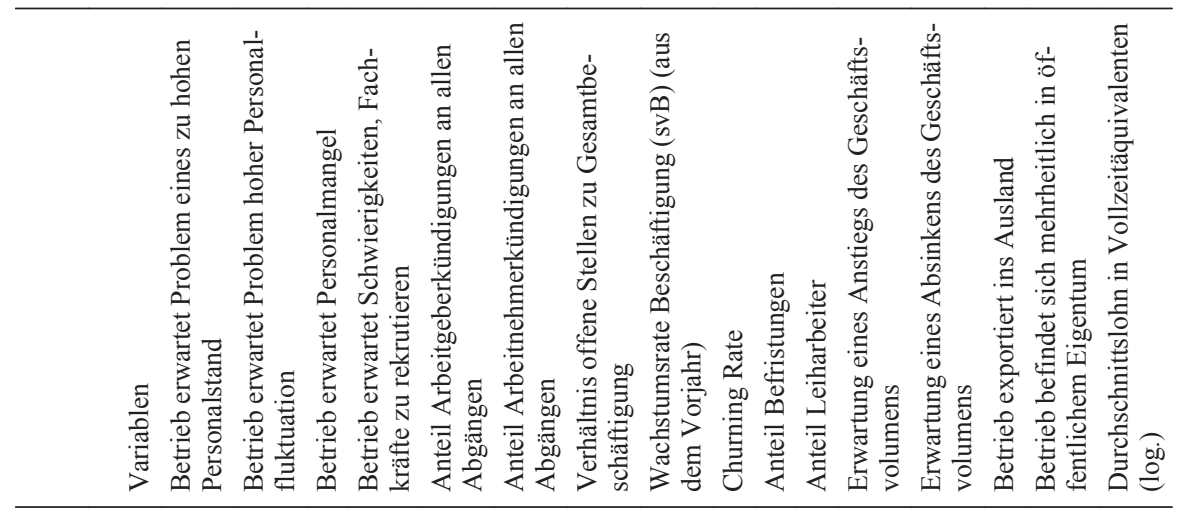




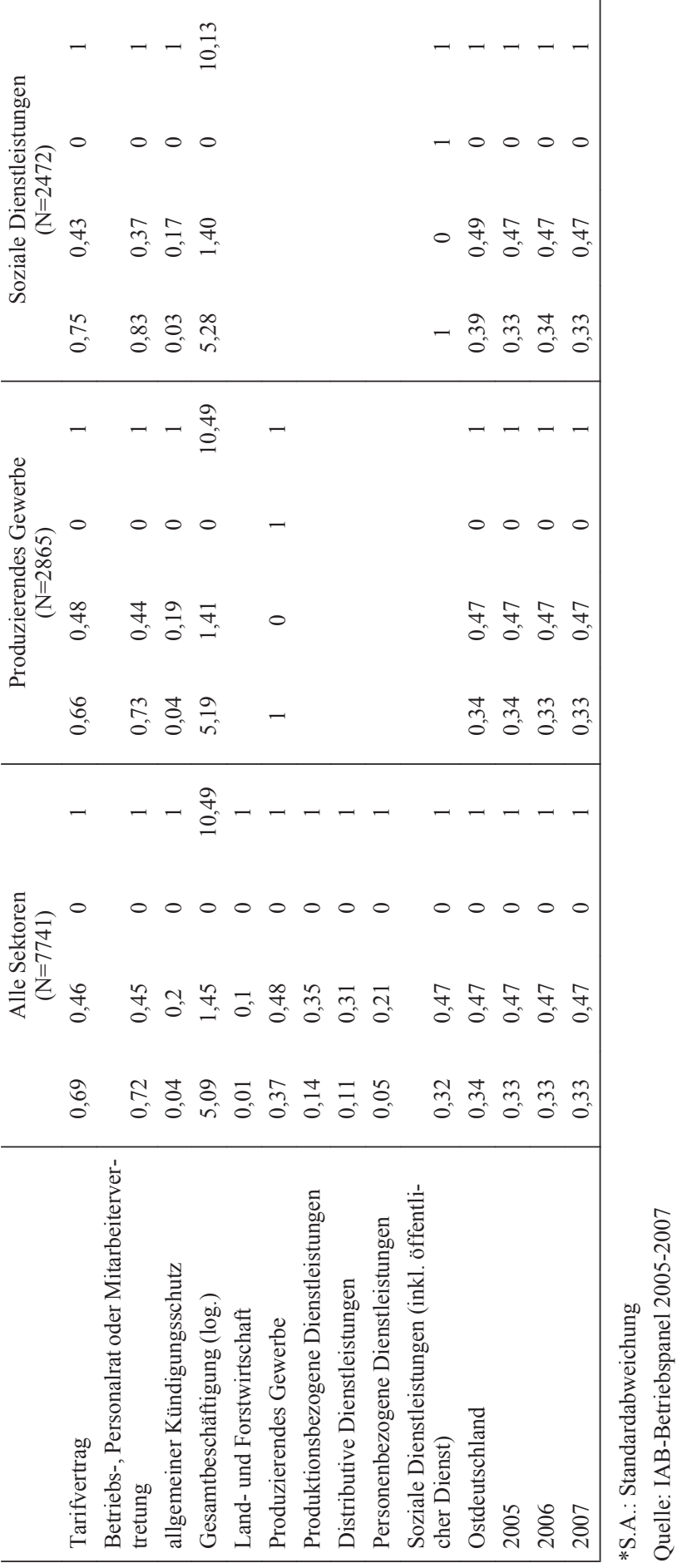

